\title{
Demokratiebarometer: ein neues Instrument zur Messung von Demokratiequalität
}

\author{
Marc Bühlmann • Wolfgang Merkel • Lisa Müller · Heiko Giebler • \\ Bernhard Weßels
}

Zusammenfassung: Ziel dieses Artikels ist die Präsentation eines neuen Demokratieindex - des Demokratiebarometers. Das Demokratiebarometer versucht, die konzeptionellen und methodologischen Schwächen bisheriger Demokratiemaße zu überwinden, um so die Qualitätsunterschiede von etablierten Demokratien messen und analysieren zu können. Der Index basiert auf einem ausdifferenzierten Demokratiekonzept, aus dem in mehreren transparenten Schritten die Messindikatoren abgeleitet werden: Aus den drei konstituierenden Prinzipien Freiheit, Gleichheit und Kontrolle werden zunächst neun grundlegende Funktionen deduziert, aus denen dann Komponenten und daraus wiederum Subkomponenten und schließlich Indikatoren abgeleitet werden. Dieses Konzept wird in einem ersten Schritt dargelegt. Danach werden die methodologischen Grundlagen - die Messung und Aggregierung - des Demokratiebarometers erläutert. Die Präsentation erster Resultate sowie die Ergebnisse verschiedener Validitätstests zeigen schließlich die Plausibilität und das Potenzial dieses neuen Messinstruments auf.

Schlüsselwörter: Demokratiemessung · Demokratiequalität

\section{Democracy Barometer: a new instrument to measure the quality of democracy}

\begin{abstract}
In this contribution we present a new instrument to assess the quality of democracythe Democracy Barometer. This measure aims at overcoming the conceptual and methodological shortcomings of previous indices and to describe and compare the differences in the democratic quality of established democracies. The Democracy Barometer is based on a comprehensive concept of democracy which is used for a stepwise deduction of measures and indicators on different levels of abstraction. Starting with three principles, freedom, equality and control, we deduce nine basic functions. Every function is further disaggregated into components, which are measured by several sub-components and indicators. In the first part of our contribution, we present the un-
\end{abstract}

Online publiziert: 27.07 .2012

(C) VS Verlag für Sozialwissenschaften 2012

M. Bühlmann $(\bowtie)$

Institut für Politikwissenschaft, Universität Bern, Bern, Schweiz

E-Mail: marc.buehlmann@ipw.unibe.ch

W. Merkel · H. Giebler · B. Weßels

Wissenschaftszentrum Berlin, Berlin, Deutschland

L. Müller

Zentrum für Demokratie Aarau (ZDA), NCCR Democracy Universität Zürich, Zürich, Schweiz 
derlying concept of democracy as well as the aforementioned process of deduction. In the second part, we focus on the methodology: the choice of indicators and scales as well as the rules of aggregation. Finally, first results and several tests for validation, which underline the potential of our new instrument for future comparative analyses, are presented.

Keywords: Measuring democracy . Quality of democracy

\section{Einleitung}

In der empirischen Demokratieforschung findet seit einiger Zeit eine Neuorientierung hinsichtlich des Forschungsgegenstands statt. Es stellt sich nicht mehr primär die Frage, ob ein politisches System eine Demokratie ist oder nicht. Vielmehr liegt das wissenschaftliche Interesse zusehends auf der Bestimmung des Demokratiegehaltes bereits etablierter demokratischer Systeme (Altman und Pérez-Liñán 2002; Berg-Schlosser 2004a, b; Bühlmann et al. 2008; Diamond und Morlino 2004; Morlino 2004a, b; Plattner 2004). Allerdings finden sich bisher kaum Indizes, mit welchen Demokratiequalität gemessen werden kann. Bisherige, etablierte Demokratiemasse wie etwa Freedom House, der Vanhanen- oder der Polity-Index (Gastil 1990; Jaggers und Gurr 1995; Vanhanen 1997, 2000, 2003) eignen sich zwar gut für die Beschreibung der Entwicklung von und der Unterschiede zwischen Demokratien und Autokratien. Sie sind aber zu wenig differenziert und valide, um feine Unterschiede zwischen etablierten Demokratien zu erfassen. So weisen etwa sowohl Freedom House wie auch Polity im Jahr 2005 für Italien unter Berlusconi oder die USA unter Georg W. Bush die gleichen Maximalwerte auf wie für Finnland unter Matti Vanhanen, obwohl der geneigte Leser die Qualität dieser drei Länder zumindest intuitiv als unterschiedlich beurteilen dürfte.

Die Schwäche bisheriger Demokratieindizes ist insbesondere dem Umstand geschuldet, dass diese auf einer (zu) minimalistischen Demokratiekonzeption basieren. Um das Phänomen ,Demokratie‘ jedoch in seiner ganzen Komplexität erfassen zu können, muss eine Demokratiequalitätsmessung auf einem breiten Demokratiekonzept fußen. Darüber hinaus weisen bisherige Indizes zahlreiche methodische Mängel auf (Müller und Pickel 2007; Munck und Verkuilen 2002). Im vorliegenden Beitrag beschreiben wir ein Instrument, das sich der Erfüllung der Ansprüche, welche an Demokratiemaße gestellt werden, annähert: das Demokratiebarometer bezweckt explizit die Messung der Qualität von etablierten Demokratien. Es stützt sich auf eine Demokratiekonzeption mittlerer Reichweite, die sowohl liberale als auch partizipatorische Demokratieentwürfe vereint, aber durch eine systematische und schrittweise Deduktion die zentralen Elemente einer Demokratie bestimmt und transparent misst. Dadurch ist das Demokratiebarometer außerordentlich anschlussfähig. Institutionelle Neuerungen oder theoriegestützte Erweiterungen können - vorausgesetzt die Datenverfügbarkeit ist gegeben - relativ einfach nachträglich eingebaut werden. Zudem beruht die Messung der Demokratiequalität, also die Auswahl der Indikatoren, die Bestimmung des Messniveaus und die Art der Aggregierung der Daten, auf ausgewiesenen theoretischen Überlegungen und empirischen Tests.

In diesem Beitrag werden neben der detaillierten Beschreibung der konzeptionellen Basis des Demokratiebarometers (Abschn. 2) und der Erörterung der Messung (Abschn. 3) erste Resultate für 30 Länder präsentiert (Abschn.4). Darüber hinaus wird in Abschn. 5 
mit verschiedenen Tests eruiert, wie valide und reliabel das neue Instrument ist. Eine Diskussion über die Möglichkeiten und Grenzen des Demokratiebarometers rundet den Beitrag ab. ${ }^{1}$

\section{Die Konzeptualisierung des Demokratiebarometers}

In der einschlägigen Literatur gibt es unzählige Definitionen von Demokratie. Das Phänomen ,Demokratie“ ist komplex, so dass kaum Einigkeit darüber herrscht, welches dessen konstituierende Elemente sein sollen. Hilfreich sind deshalb Versuche, die verschiedenen Vorschläge zu systematisieren und in unterschiedliche idealtypische Modelle einzuteilen (z.B. Held 2006; Merkel 2010; Schmidt 2000). Dabei lassen sich drei hauptsächliche Modelle ausmachen, die sich anhand ihrer konzeptuellen Komplexität, ihrer Ansprüche und Ziele unterschieden lassen:

1. Aus einer minimalistischen Perspektive wird Demokratie als Mittel betrachtet, mit welchem die Freiheitsrechte der Bürgerinnen und Bürger vor Übergriffen durch den Staat geschützt werden. In freien und fairen Wahlen werden Interessen durch die Bestimmung fähiger, politischer Eliten artikuliert. Eine Einmischung der Bürgerschaft in politische Belange ausserhalb regelmässig stattfindender Wahlen ist nicht vorgesehen. Klassische Vertreter dieses liberalen, minimalistischen Models sind etwa Locke (1974 [1689]), Montesquieu (1965 [1748]), Mill (1991 [1861]) und Tocqueville (1997 [1835]). Das Modell findet moderne Erweiterungen in elitären (Weber 1988 [1921]) wie in pluralistischen Demokratieentwürfen (Dahl 1956; Fraenkel 1962, 1991; Truman 1971 [1951]). Schumpeters (1950) realistische Demokratietheorie kann als prononcierteste Form minimalistischer Modelle betrachtet werden.

2. Anspruchsvoller ist die partizipatorische Konzeption von Demokratie. Politische Beteiligung und Mitsprache werden in diesem Modell als Wert an sich und als Kern von Demokratie betrachtet, da sie die individuelle Selbstentfaltung, politisches Interesse und die gesellschaftliche Integration fördern. Den Bürgerinnen und Bürgern müssen möglichst viele Opportunitäten für möglichst unmittelbare Partizipation geboten werden. Darüber hinaus sollen gemeinsam gefasste politische Entscheide Produkt möglichst deliberativer Prozesse darstellen. Der partizipatorische Typus wurzelt in einer Demokratie nach athenischem Vorbild (Fenske et al. 1994, S.37) und dem klassischen Republikanismus von Rousseau (1977 [1762]) sowie in neuerer Zeit in den Ideen der partizipatorischen Demokratie (Barber 1984; Pateman 1970) und teilweise der deliberativen Demokratietheorie (Cohen und Fung 2004; Fishkin 1991; Habermas 1992; Offe und Preuss 1991; Warren 1996).

1 Das Demokratiebarometer ist ein vom schweizerischen Nationalfonds unterstütztes Forschungsprojekt, das im Rahmen des NCCR Democracy durchgeführt wird. Gedankt sei an dieser Stelle dem Zentrum für Demokratie Aarau sowie dem Wissenschaftszentrum Berlin für die Unterstützung. Dank gebührt nicht zuletzt auch den zahlreichen Mitarbeiterinnen und Mitarbeitern, ohne die das Demokratiebarometer nicht entstanden wäre: Stefani Gerber, Miriam Hänni, Ruth Kunz, Lisa Schädel, Dag Tanneberg, Max Schubiger, Isabel Vollenweider und David Zumbach. 
3. Der dritte Typus nimmt Teile der minimalistischen und partizipatorischen Modelle auf und erweitert sie mit sozialen Voraussetzungen, welche für freie und faire Partizipation erfüllt sein müssen. Er bezieht zusätzlich Politikergebnisse in seine Definition mit ein, wenn sie Bereiche der sozialen Gerechtigkeit, im Sinne einer sozialen Demokratie, berühren. Die Etablierung sozialer Rechte und die Reduzierung sozioökonomischer Ungleichheiten werden als inhärente demokratische Ziele betrachtet. Demokratien müssen daher entsprechende Leistungen erbringen (vgl. Heller 1934, 1971; Miller et al. 1967; Meyer 2005; Rawls 1971 oder Sen 1979, 1996, 1997).

Bisherige Demokratieindizes basieren entweder auf minimalistischen Demokratieentwürfen ${ }^{2}$ oder relativ anspruchslosen Beurteilungskriterien. ${ }^{3}$ Damit lassen sich zwar durchaus demokratische von nicht-demokratischen Regimen unterscheiden. Für die Bestimmung feiner Unterschiede hinsichtlich der Demokratiequalität eines Landes muss das Phänomen ,Demokratie“ allerdings möglichst in seiner ganzen Komplexität erfasst werden. Das Demokratiebarometer löst sich deshalb von rein minimalistischen Demokratievorstellungen und basiert auf einer Demokratiekonzeption mittlerer Reichweite. Es verbindet Elemente des liberalen und des partizipatorischen Modells und beleuchtet somit den Gegenstand ,Demokratie“ - entsprechend der Popper'schen Schweinwerfermetapher (1992 [1957]) - gleichzeitig aus unterschiedlichen Perspektiven.

Dabei werden jedoch Forderungen nach einem spezifischen demokratischen Output im Sinne des oben beschriebenen dritten Typus aus zwei Gründen abgelehnt. Erstens werden Outputs als genuin politische Entscheidungen betrachtet, die durch Demokratie als Mittel hervorgebracht werden sollten, nicht aber Bestandteil von Demokratie sind. Zweitens sind Politikergebnisse (im Sinne von Outcomes) nicht nur unmittelbares Resultat von demokratischen Entscheidungen, denn auch ökonomische oder soziostrukturelle Faktoren beeinflussen die Umsetzung politischer Entscheidungen.

Auf der Basis dieses Konzepts wird in der Folge über mehrere miteinander verknüpfte Schritte ein theoretisch fundiertes Messinstrument der Demokratie entwickelt. Ausgangspunkt bildet die Prämisse, dass demokratische Systeme eine Balance zwischen den interdependenten Werten ,Freiheit' und ,Gleichheit' herzustellen versuchen und sich dazu einer dritten demokratieinhärenten Dimension bedienen: Kontrolle. Kontrolle hat darüber hinaus einen prinzipiellen Wert für die Demokratie. Herrschaftskontrolle ist einer der kardinalen Unterschiede, der die Demokratie von der Autokratie mit ihrer tendenziell unkontrollierten Machtausübung unterscheidet. Mit Hilfe dieser drei Grundprinzipien werden dann in einem zweiten Schritt neun Funktionen hergeleitet, von deren Erfüllungsgrad die Qualität einer Demokratie abhängt. Dieser Erfüllungsgrad wird anhand verschiedener

2 Dazu gehören nicht nur der Polity- oder der Vanhanen-Index, sondern auch die Arbeiten von Hadenius (1992); Arat (1991); Coppedge und Reinicke (1990); Bollen (1990); Gasiorowski (1990) und Alvarez et al. (1996), die implizit oder explizit auf dem empirisch minimalistischen Demokratieentwurf von Dahl (1971) basieren, wie er in dessen Polyarchiemessung Verwendung findet (vgl. dazu Lauth 2004; Müller und Pickel 2007).

3 Dies ist etwa der Fall bei Freedom House, das in seiner Demokratiedefinition sichtbar über Dahl hinausgeht, aber bei der Kriterienbeurteilung so anspruchslos ist, dass sich auch über Jahrzehnte hinweg bei einer Gruppe von mehr als 30 Ländern (etablierte Demokratien) nahezu keine Varianz ergibt. 
Komponenten bestimmt, die ihrerseits durch Subkomponenten und geeignete Indikatoren gemessen werden.

\subsection{Demokratieprinzipien Freiheit, Gleichheit und Kontrolle}

Die Messung von Demokratiequalität sollte auf einer theoretisch solide abgestützten Demokratiedefinition beruhen. So lapidar dies klingt, so selten wird eine stringente Diskussion und Begründung des Messkonzepts in der zeitgenössischen Demokratiemessung tatsächlich vorgenommen (Berg-Schlosser 1999; Collier und Levitsky 1997; Munck und Verkuilen 2002). Das Demokratiebarometer argumentiert, dass Demokratie auf drei fundamentalen Prinzipien beruht: Freiheit, Gleichheit und Kontrolle.

\subsubsection{Freiheit}

Freiheit - verstanden als negative Freiheit - bedeutet vorerst Schutz eines Individuums vor den illegitimen Eingriffen dritter: dieser Schutz besteht gegenüber dritten Privatpersonen aber insbesondere gegenüber dem Staat. Die historisch bedeutsamsten Aspekte dieser Abwehrrechte sind das Recht auf Integrität von Leib und Leben, Meinungs- und Religionsfreiheit und - durchaus umstrittener - das Recht auf Eigentum. Die Liste der Freiheiten ist mit der Zeit angewachsen (z. B. Meinungsfreiheit, Versammlungsfreiheit, Informationsfreiheit; vgl. Merkel et al. 2003), und deren rechtsstaatlich garantierter Schutz gilt mittlerweile als Minimalbedingung für demokratische Regime: Demokratie ohne Freiheit wird als „contradiction in terms“ (Beetham 2004, S. 62) betrachtet. Von den negativen Abwehrrechten sind politische wie soziale Beteiligungsrechte abzugrenzen. Politische Beteiligungsrechte sind in der Demokratie dem Gleichheitsgebot unterworfen (Rawls 1971), während soziale Beteiligungsrechte unter Ressourcenvorbehalt (Höffe 1999, S. 75) stehen. Erstere fassen wir unter ,Gleichheit', während wir letztere über unser Demokratiekonzept mittlerer Reichweite aus der Demokratiedefinition ausgeschlossen haben.

Verbriefte und durchgesetzte Freiheitsrechte sind eine Voraussetzung der Demokratie. Sind sie nicht garantiert, können die Bürger ihre politischen Beteiligungsrechte nicht hinreichend geschützt wahrnehmen. Die Garantie solcher Rechte kann keineswegs alleine über die Volkssouveränität realisiert werden, sondern bedarf der Sicherung durch den Rechtsstaat. Habermas hat diese funktionale Interdependenz die Gleichursprünglichkeit von Volkssouveränität und Menschenrechten, von Demokratie und Rechtsstaat genannt (Habermas 1992; Meyer 2009). Zu den für die Demokratie unverzichtbaren Bürgerrechten zählen aber nicht nur Integrität von Leib und Leben, Freiheit und Schutz vor willkürlicher Verhaftung und Eigentum (Locke 1974 [1689]). Auch Versammlungs- und Organisationsrechte sowie Meinungs- und Medienfreiheit, die eine lebendige Zivilgesellschaft ermöglichen, gehören zu den demokratischen Basisrechten (Linz und Stepan 1996). Effektive Meinungsfreiheit hängt allerdings ab von den „Bedingungen, unter denen der Bürger Informationen erhält"“ (Sartori 2006, S. 94). Demokratie kann also nur dann substantiell freiheitlich sein, wenn der freie Informationsfluss gewährleistet und eine grundlegende Möglichkeitsbedingung demokratischer Öffentlichkeit unverbrüchlich geschützt ist. 
Historisch wie funktional ist Freiheit eng verknüpft mit Volkssouveränität. Wie die politischen Kämpfe des 19. Jahrhunderts gezeigt haben, kann eine tatsächliche Garantie bürgerlicher Freiheitsrechte nur dort funktionieren, wo politische Teilhaberechte für alle Bürger gewährleistet sind (Habermas 1992; Meyer 2005). In diesem Sinne hängen Freiheit und politische Gleichheit eng zusammen.

\subsubsection{Gleichheit}

Obwohl schon in der griechischen Stoa die natürliche Gleichheit aller rationalen Wesen betont wird und später im Neuen Testament, dem Talmud und im Islam die Gleichheit der Menschen vor Gott zum Prinzip erhoben wird, gelingt der Idee der politischen Gleichheit erst in den Vertragstheorien der Neuzeit der Durchbruch (Gosepath 2007, S. 4). Von Hobbes über Locke bis Rousseau ist die Gleichheit der Menschen im Naturzustand die Ausgangsfigur herrschaftspolitischen Denkens. In Kants Moral- und Rechtsphilosophie führen die Reflexionen über Autonomie und Selbstgesetzgebung zu der gleichen Freiheit für alle rationalen Wesen (Kant 1902 [1785]). In der Französischen Revolution wurde Gleichheit, neben Freiheit und Brüderlichkeit zur Grundlage der Déclaration des droits de l'homme et du citoyen von 1789.

In der Moderne sind Gleichheit und Freiheit in der philosophischen aber insbesondere der politischen Moderne unauflösbar verknüpft. Freiheit verlangt zwingend eine Gleichheitskomponente, ohne die eine gleiche individuelle Autonomie nicht zu haben ist. Wie in der Kantischen Republik müssen sich die Bürger in der modernen Demokratie die gleiche Autonomie zubilligen (Nida-Rümelin 2006, S. 125; 144-145).

Mindestens zwei Gründe sprechen dafür, politische Gleichheit als fundamentales Prinzip einer Demokratie zu bezeichnen (Dahl 1998, 2006). Aus einer normativ-moralischen Perspektive gehen moderne Gesellschaften erstens vom Standpunkt aus, dass keine objektive Bewertungsgrundlage dafür existieren kann, ob der Lebensentwurf von Individuum A besser oder schlechter sei als jener von Individuum B. Zweitens: „no persons are so definitely better qualified than others to govern that they should be entrusted with complete and final authority over the government of the state" (Dahl 1998, S. 75).

Gleichheit - hier immer verstanden als politische Gleichheit - bedeutet v. a. Gleichbehandlung aller Individuen im politischen Prozess. Allen Bürgerinnen und Bürger müssen die gleichen Rechte zur Beeinflussung demokratischer Entscheidungen eingeräumt werden (Dahl 1976). Darüber hinaus muss die gesamte Bürgerschaft über gleiche Zugangschancen zu politischer Macht verfügen (Böckenförde 1991; Vossenkuhl 1997; Saward 1998; Lauth 2004).

Aus dem Prinzip ,Politische Gleichheit‘ erwächst die Kompetenz für demokratische Selbstregierung, die auf den Schultern möglichst aller Bürger ruhen muss. Damit logisch verbunden ist die Forderung nach einer möglichst breiten Inklusion aller Gesellschaftsmitglieder auch und insbesondere durch eine möglichst umfassende politische Partizipation. Ein Postulat, dass trotz seiner zwingenden Logik v.a. in der angelsächsischen Demokratietheorie vernachlässigt oder bestritten wird.

Wir argumentieren dagegen, dass politische Gleichheit insbesondere dann effektiv ist, wenn eine gleichberechtigte Einbindung und Berücksichtigung möglichst aller individuellen Präferenzen gesichert wird. Die gleichberechtigte Einbindung von Präferenzen ist 
dabei nicht nur abhängig von rechtsstaatlichem Schutz, sondern bedingt aus einer pluralistischen Perspektive (Fraenkel 1991) auch eine gut organisierte, aktive Öffentlichkeit, welche dazu beiträgt, Interessen zu bündeln und zu aggregieren. Zentral sind darüber hinaus in einer repräsentativen Demokratie freie, faire und kompetitive Wahlen, welche die gleichberechtigte Einbindung von Interessen zulassen. Politische Partizipation, nicht nur konventioneller sondern auch unkonventioneller Art, sollte zudem möglichst gleich wahrgenommen werden können, um zu verhindern, dass gewisse Gesellschaftsgruppen systematisch vom politischen Prozess ausgeschlossen werden (Teorell et al. 2007; Lijphart 1997).

Auch wenn freie, faire und kompetitive Wahlen garantieren können, dass Interessen gleichberechtigt in den politischen Prozess eingespeist werden, bedeutet dies noch nicht, dass Präferenzen auch gleichberechtigt zu politischen Outputs verarbeitet werden. Eine gleichberechtigte Einbindung aller Präferenzen setzt deshalb auch eine möglichst adäquate deskriptive und substantielle politische Repräsentation voraus. Eine wichtige Voraussetzung für eine gleichberechtigte Wahrnehmung von politischen Rechten sowie für eine responsive Einbindung aller Präferenzen ist schliesslich, dass der politische Entscheidungsprozess für alle gleichermassen sichtbar und durch eine hohe Transparenz gekennzeichnet ist (Stiglitz 1999).

\subsubsection{Kontrolle}

Das Streben nach Freiheit und Gleichheit gilt als fundamentale und treibende Kraft hinter der Entwicklung von modernen repräsentativen Demokratien. Allerdings sind die beiden Prinzipien nicht nur gleichursprünglich komplementär, sondern sie stehen in einem interdependenten Verhältnis zueinander (Talmon 1960 und bereits Tocqueville 1997 [1835]). Die Beziehung der beiden Prinzipien wird hier als dynamisches Gleichgewicht gedacht, wobei die Etablierung einer optimalen Balance eine Kernherausforderung einer Demokratie darstellt. Ein drittes Prinzip dient dazu, das labile Gleichgewicht zu halten: Kontrolle oder, in anderen Worten, Machtbeschränkungen der politischen Repräsentanten. Dies geschieht sowohl vertikal durch die Bürger als horizontal durch konstitutionelle wie institutionelle Kontrollmechanismen, mit deren Hilfe die demokratischen Gewalten sich gegenseitig überwachen und beschränken.

Die horizontale Kontrolle funktioniert im Sinne der klassischen Gewaltentrennung als Netzwerk relativ autonomer Institutionen, mit deren Hilfe gewählte Autoritäten kontrolliert werden bzw. sich gegenseitig in ihrer Macht ausbalancieren (O’Donnell 1994). Diese Funktion widerspiegelt somit eine der ältesten Forderungen in der Theorie der repräsentativen Demokratie, nämlich die Idee, dass die verschiedenen Gewalten in einem Staat durch Beaufsichtigung gegenseitig Machtmissbrauch verhindern (Locke 1689; Montesquieu 1748).

Ein zentraler Anspruch an die Demokratie ist, dass Bürgerinnen und Bürger einen grossen Spielraum für die Gestaltung gesellschaftlicher Verhältnisse haben: sie müssen also ,selbst entscheiden [können], welche Bilanz sie im Falle der unvermeidlichen trade off's zwischen Freiheit und Gleichheit ziehen wollen“" (Meyer 2009, S. 59). Diese Entscheidung wird in repräsentativen Demokratien primär über Wahlen getroffen. Wahlen garantieren vertikale Kontrolle, indem sie den Bürgerinnen und Bürgern erlauben, die 
politischen Repräsentanten wiederzuwählen oder zu ersetzen (Manin et al. 1999). Wirksame Wahlen müssen möglichst frei und fair und insbesondere kompetitiv sein. Entsprechend erfordert eine Demokratie einen hohen Grad an Wettbewerb bei der Besetzung von politischen Ämtern (Bartolini 1999, 2000; Dahl 1971). Erst kompetitive und offene Wahlen ermöglichen eine tatsächliche Auswahl und zwingen so die Amtsträger zu responsivem Verhalten.

Wahlen können allerdings nur dann effektiv im Sinne von responsiv sein, wenn es der Regierung überhaupt möglich ist, Entscheidungen zu fällen und umzusetzen. Die Idee der vertikalen Kontrolle würde korrumpiert, wenn Bürgerinnen und Bürger zwar Personal bestellen würden, das verspricht, im Interesse der Wählerinnen und Wähler zu handeln, dieses Versprechen dann aber nicht umsetzen kann, weil es nicht über genügend Kapazitäten verfügt. Die Regierung muss also über eine hinreichende Handlungsfähigkeit verfügen. Nur wenn es möglich ist, aus den Wählerinteressen auch politische Entscheidungen zu formen, können Wahlen effektiv und Repräsentation adäquat sein (Alonso et al. 2010). Regierungen benötigen eine gewisse Kontrolle über das politische Geschehen bzw. eine ausreichende Handlungsfähigkeit, um demokratisch gefällte Entscheidungen effizient umsetzen zu können. Wenn Demokratie Selbstbestimmung durch kollektive Entscheidungen bedeutet, muss es die demokratisch bestimmte Regierung sein, welche diese Entscheidungen letztendlich umsetzt. Sie darf dabei nur durch neue, demokratisch legitimierte Entscheidungen oder durch rechtsstaatliche Barrieren eingeschränkt sein (Etzioni 1968), nicht jedoch durch außerkonstitutionelle private, politische, religiöse oder militärische Akteure. Die Durchsetzungsfähigkeit von demokratisch legitimierten Entscheidungen durch eine demokratisch gewählte Regierung ist deshalb ein entscheidender Bestandteil demokratischer Kontrolle und demokratischer Qualität.

Aus einer partizipatorischen Perspektive sollte die vertikale Kontrolle zudem auch zwischen den Wahlen ausgeübt werden können. Eine zentrale Rolle spielt dabei die Aufdeckung und Diskussion von Missständen durch eine aktive Öffentlichkeit (vgl. dazu bereits Tocqueville 1997 [1835]) in Form einer lebendigen Zivilgesellschaft und in Form von freien Medien. Diese Kontrolle ist allerdings wiederum nur möglich, wenn die Transparenz politischer Prozesse gewährleistet ist und wenn die entsprechenden individuellen Freiheiten garantiert sind.

Zusammenfassend definieren wir Freiheit, Gleichheit und Kontrolle als fundamentale Kernprinzipien einer Demokratie. Damit ein Regime als Demokratie bezeichnet werden kann, muss es Freiheit und Gleichheit garantieren und schützen. Darüber hinaus muss es die Interdependenzen dieser beiden Prinzipien vermittels vertikaler und horizontaler Kontrolle balancieren und optimieren. Kontrolle ist freilich mehr als lediglich ein Instrument zur optimalen Balance, es muss als gleichursprüngliches Prinzip von Demokratie verstanden werden.

\subsection{Die neun Funktionen von Demokratie und ihre Komponenten}

Um Freiheit, Gleichheit und Kontrolle zu garantieren und funktional zu sichern, müssen demokratische Systeme unterschiedliche Funktionen erfüllen, welche sich theoretisch aus den Prinzipien herleiten lassen. Die Ableitungsrichtung, die wir auf der Prinzipienebene 
Abb. 1: DemokratiebarometerKonzeptbaum (Ausschnitt)

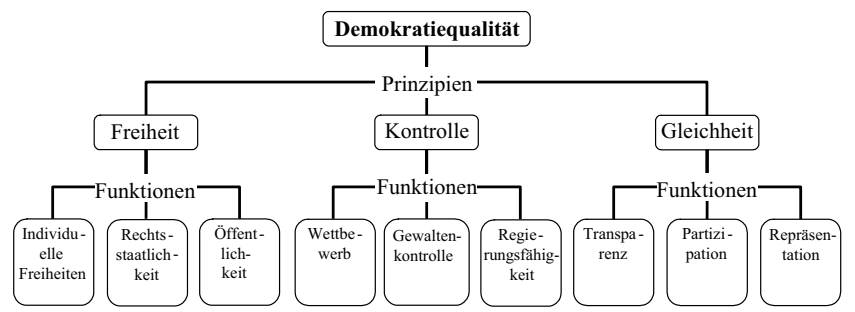

herausgearbeitet haben und unten auf der Funktionsebene noch weiter ausdifferenzieren, wird in Abb. 1 noch einmal verdeutlicht.

Im Demokratiebarometer wird nun Demokratiequalität anhand des Erfüllungsgrads dieser neun Funktionen gemessen: je höher der Erfüllungsgrad der einzelnen Funktionen ist, desto besser ist die Demokratiequalität. Eine simultane Maximierung der neun Funktionen ist theoretisch zwar wünschenswert, realiter jedoch kaum möglich, da vermutet werden kann, dass die einzelnen Funktionen in zielinkongruenter Spannung zueinander stehen. Dies ist bereits im Spannungsverhältnis von Freiheit und Gleichheit angelegt. Verschiedene Demokratien - verstanden als Systeme, die durch politische wie auch gesellschaftliche Kräfte immer wieder neu ausgehandelt werden - werden deshalb die neun Funktionen unterschiedlich gewichten. Es kann allerdings davon ausgegangen werden, dass es einzelnen Demokratien besser gelingt, die neun Funktionen in ein optimales Gleichgewicht zu bringen, und diese deshalb eine insgesamt höhere Demokratiequalität erreichen als andere. Wir erwarten entsprechend unterschiedliche empirische Realisierungen von Demokratie. Ein Hauptziel des Demokratiebarometers ist es denn auch, diese ,varieties of democracies' zu analysieren und zu bewerten.

In der Folge werden die neun Funktionen genauer definiert und auf eine spezifischere, messbare Ebene heruntergebrochen. Wir gehen kurz auf jede Funktion entsprechend der Anordnung in Abb. 1 ein. Funktionen werden dabei durch jeweils zwei Komponenten näher bestimmt, die ihrerseits durch Subkomponenten definiert werden. Die Subkomponenten werden dann mit Hilfe verschiedener Indikatoren gemessen. Es wird dabei unterschieden zwischen Subkomponenten, deren Indikatoren verfasste Regeln messen und Subkomponenten, deren Indikatoren die Verfassungswirklichkeit bestimmen.

\subsubsection{Individuelle Freiheiten}

Die Existenz und Garantie von individuellen Freiheiten in Demokratien ist eine, wenn nicht die grundlegende Voraussetzung für die demokratische Selbst- und Mitbestimmung der Bürger. Freiheit ist als die Unverletzlichkeit der persönlichen Sphäre zu verstehen. Insofern lassen sich die ,negativen Freiheitsrechte' als unverbrüchlicher Schutz gegen die Intervention dritter Privatpersonen aber v. a. auch vor dem Staat verstehen (Höffe 1999, S. 66; Berlin 2006). Zu den individuellen Freiheiten gehört zuallererst das Recht auf körperliche Unversehrtheit. Diese erste Komponente umfasst drei Subkomponenten, von denen die erste durch die Existenz von Verfassungsgarantien und die Ratifizierung wichtiger internationaler Menschenrechtskonventionen die staatliche Kultur im Umgang mit Folter erfasst (Camp Keith 2002; Cingranelli und Richards 1999; O’Donnell 2004; Saward 1994). Verfassungswirklichkeit wird durch die zweite Subkomponente anhand 
von Verstössen gegen das Recht auf körperliche Unversehrtheit der Bürger durch den Staat bestimmt. Die dritte Subkomponente schließlich basiert auf der Idee, dass staatlicher Rechtsschutz nur dann effektiv sein kann, wenn die Bürgerschaft selbst bereit ist, das gegenseitige Recht auf Unversehrtheit anzuerkennen (Beetham 2004, S. 72). Als Indizien gegen eine breite Akzeptanz werden die Mordrate und die Anzahl politischer Aktionen, in denen Gewalt angewendet wird, herangezogen.

Die zweite Komponente beschreibt einen weiteren wichtigen Aspekt individueller Freiheiten: das Recht auf Selbstbestimmung. Dazu gehört einerseits das Recht auf eine freie Lebensgestaltung in Form von Religions- und Bewegungsfreiheit. Andererseits müssen Eigentumsrechte effektiv geschützt sein. Auch hier wird unterschieden zwischen verfassungsmässiger Garantie dieser Rechte und effektiver Verfassungswirklichkeit.

\subsubsection{Rechtsstaatlichkeit}

Ein zentraler Garant für die Einhaltung demokratischer Prinzipien und unverzichtbarer Grundrechte ist keineswegs die vertikale Kontrolle durch die Bürger allein, sondern der Rechtsstaat. Er sichert die Freiheitsrechte und politischen Rechte, ohne die Partizipation auch nur an freien Wahlen nicht zu denken ist. Niemand hat eindrucksvoller auf die Rolle der rechtsstaatlichen Sicherung von demokratischen Beteiligungsrechten hingewiesen als Habermas (1992), als er von der Gleichursprünglichkeit von Freiheits- und Beteiligungsrechten gesprochen hat. Beide Rechte verweisen funktional aufeinander, sonst können sie keinen Bestand haben. Insofern lässt sich der Rechtsstaat dem Prinzip der Freiheit zuordnen, wiewohl er auch Funktionen der Kontrolle und der Gleichheitssicherung erfüllt.

Dies bedeutet erstens, dass alle Bürger eines Staates vor dem Gesetz gleich sind und gleich behandelt werden müssen (Beetham 2004; Esquith 1999; Rawls 1971). Entsprechend ist Gleichheit vor dem Gesetz die erste Komponente von Rechtsstaatlichkeit. Sie wird mit Hilfe von drei Subkomponenten untersucht. Die erste beinhaltet die verfassungsmäßig garantierte Unparteilichkeit von Gerichten. Die beiden anderen Subkomponenten bestimmen die effektive Unabhängigkeit der Judikative bzw. die effektive richterliche Objektivität. Gleichheit vor dem Gesetz kann nur verwirklicht sein, wenn Gerichte von spezifischen politischen Interessen möglichst unbeeinflusst bleiben und nicht manipulierbar sind (O’Donnell 2004).

Die zweite Komponente von Rechtsstaatlichkeit soll die Qualität der Justiz bestimmen. Auch hier werden drei Subkomponenten herangezogen. Erstens sichern verfasste Regeln die Professionalität der Judikative (berufliche Qualifikationen, Amtsdauer) (Camp Keith 2002; La Porta et al. 2004). Daneben kann die Legitimität der Justiz als Indiz für ihre Qualität betrachtet werden. Die Judikative bezieht im Unterschied zu den beiden anderen Gewalten ihre Legitimität nicht aus Wahlen, sondern diese basiert auf der demokratisch verabschiedeten Verfassung und dem anhaltenden Vertrauen der Bevölkerung in das Justizsystem (Bühlmann und Kunz 2011; Gibson 2006) und in die Inhaber des rechtsstaatlichen Gewaltmonopols. 


\subsection{3 Öffentlichkeit}

Freiheit beruht aber nicht nur auf Rechtsstaatlichkeit und der Garantie individueller Freiheiten, sondern hat eine wichtige kollektive Bestimmung: Freiheit bedeutet auch, sich mit Anderen auszutauschen, sie von der eigenen Meinung zu überzeugen und für eine Sache zu mobilisieren versuchen (Beetham 2004, S. 62). In einem emphatischen Sinne ist dies bei Habermas in der Nachfolge von Kant die Sphäre der Vermittlung von Politik und Moral (Habermas 1990 [1962], S. 178). Die Demokratie-Funktion ,Öffentlichkeit‘ wird im Demokratiebarometer über die zwei Komponenten ,Vereinigungsfreiheit ${ }^{\star}$ und ,Meinungsfreiheit' erfasst. Vereinigungsfreiheit muss dabei nicht nur konstitutionell abgesichert sein, sondern deren Funktionieren wird in Anlehnung an die Sozialkapitalforschung anhand der Aktivität der Zivilgesellschaft und der Öffentlichkeit bestimmt (Putnam 1993; Teorell 2003; Young 1999). Bereits Tocqueville (1997 [1835]) argumentierte, dass Organisationen, Vereine und Gewerkschaften als wichtige Schulen der Demokratie wirken. Eine hohe Organisationsdichte in politischen Interessenorganisationen wie auch in Organisationen, die öffentliche Interessen verfolgen, wird hier deshalb als Proxy für eine funktionierende, freie Artikulation von Präferenzen gedeutet. Dass diese freie Artikulation freier Assoziationen kollektiv geschieht und nicht allein im Publikum singulärer Privatleute, ist Teil des Strukturwandels der Öffentlichkeit und verleiht dieser eine höhere Wirksamkeit als sie durch einzelne Individuen je erzeugt werden könnte.

Artikulation von Interessen setzt Meinungsfreiheit voraus - die zweite Komponente. Auch Meinungsfreiheit muss wiederum in der Verfassung verankert sein. In der demokratischen Praxis wird freie Meinungsäusserung in repräsentativen Demokratien v.a. durch ein freies, vielfältiges und ausgewogenes Pressesystem gewährleistet. Demokratische Medien müssen allen kollektiven Akteuren und Bürgern ein Forum bieten, in dem ein möglichst breites Spektrum an Informationen zugänglich gemacht wird, sowie Meinungen geäußert und öffentliche Diskurse geführt werden können (Beierwaltes 2000; Graber 2003, 2004; Norris 2000). Wie frei und effektiv dies geschieht, bestimmt die Qualität der demokratischen Öffentlichkeit.

\subsubsection{Wettbewerb}

Die vertikale Kontrolle der gewählten politischen Repräsentanten wird dann gestärkt, wenn in Wahlkämpfen ein möglichst großer Wettbewerb herrscht. Bartolini $(1999,2000)$ unterscheidet vier Bereiche von Wettbewerb, wobei zwei davon - vulnerability und contestability- unserer Demokratiekonzeption mittlerer Reichweite bzw. der Funktion der vertikalen Kontrolle am besten entsprechen (Bartolini 2000, S. 61). ${ }^{4}$ Die Komponente vulnerability entspricht einerseits der Unsicherheit des Wahlresultats, welche sich durch die Knappheit eines Wahlausgangs bestimmen lässt (Bartolini 2000, S. 52; Elkins 1974; Strom 1992). Andererseits bedeutet vulnerability im Sinne von Konkurrenz eine geringe Konzentration von Sitzen auf einzelne Parteien im Parlament. Darüber hinaus wird der Grad an Konkurrenz von Wahlen aber auch durch formelle Regeln eingeschränkt: die

4 Die beiden anderen Bereiche umfassen die Elasitzität der Wählernachfrage (availability) und die Transparenz des alternativen Angebots (decidability). 
Größe der Wahlbezirke, aber auch gesetzliche Grundlagen hinsichtlich der Einteilung von Wahlbezirken (Gerrymandering) können die Konkurrenzsituation des politischen Wettbewerbs stark beeinflussen.

Die Komponente contestability bezieht sich auf die Höhe der gesetzlich vorgegebenen Hürden, die Parteien oder Kandidaten überwinden müssen, um überhaupt bei Wahlen antreten zu können. Dazu gehören administrative Zulassungsbarrieren und gesetzlich festgelegte Quoren, die mehr oder weniger hoch sein können. Effektive Eintrittschancen können hingegen mit Hilfe der effektiven Anzahl antretender Parteien, dem Verhältnis zwischen antretenden und gewählten Parteien sowie der Existenz und Gewinnchancen von kleinen Parteien eruiert werden (Bartolini 1999; Tavits 2006).

\subsubsection{Gewaltenkontrolle}

Die gegenseitige Kontrolle der demokratischen Gewalten gründet in der seit Locke (1689) und Montesquieu (1748) verbreiteten Idee des Machtausgleichs zwischen den demokratischen Gewalten. Dieser hängt erstens von der Beziehung zwischen der Exekutive und der Legislative ab. Die Machtbalance zwischen diesen beiden Gewalten sollte möglichst ausgeglichen sein. Dies bedingt, dass die Regierungs- und Oppositionskräfte im Parlament möglichst gleich stark bzw. der Sitzanteil der Regierungskräfte in der Legislative möglichst klein ist. Zudem sorgen institutionalisierte Absetzungs- bzw. Auflösungsmöglichkeiten der Regierung bzw. des Parlamentes für eine effektive wechselseitige Kontrolle der Exekutive und der Legislative (de la Porta et al. 2004). Dabei können bereits Sanktionsandrohungen im Sinne von verfassten Opportunitäten als wichtige Voraussetzung für horizontale Verantwortlichkeit betrachtet werden (Bovens 2007).

Die zweite Komponente der Funktion Gewaltenkontrolle umfasst weitere institutionelle Sicherungen: auf der einen Seite handelt es sich dabei um die Verfassungsgerichtsbarkeit bzw. die verbriefte Möglichkeit der Judikative, die Verfassungsmäßigkeit von Gesetzesvorhaben zu überprüfen. Auf der anderen Seite wird der Föderalismusgrad eines Landes als wichtige Veto- und Kontrollmöglichkeit betrachtet (Hamilton und Madison 1993 [1788]). Hier fließen - in Anlehnung an die Föderalismus- und Dezentralisierungsforschung (Steffani 1979; Schneider 2003) - sowohl der institutionalisierte Grad an Dezentralisierung wie auch die effektive subnationale fiskale Autonomie als Subkomponenten ein. Die horizontale und vertikale demokratische Gewaltenkontrolle, die in minimalistischen Demokratiedefinitionen von Schumpeter (1950) bis Dahl (1971) keinen Platz findet, betrachten wir als ein wesentliches Qualitätsmerkmal der repräsentativen Demokratie.

\subsubsection{Regierungs- und Implementierungsfähigkeit}

Eine zentrale Idee repräsentativer Demokratie ist, dass die Präferenzen des Demos gesammelt, gebündelt, artikuliert, über Wahlen aggregiert und in Parlamentsmandate übersetzt werden, deren Inhaber dann mit der politischen Entscheidungsfindung betraut sind. In einer responsiven Demokratie wird diese Kette um ein weiteres Glied verlängert, nämlich die Umsetzung von politischen Entscheidungen, welche den ursprünglich eingespeisten Präferenzen entsprechen (Powell 2004). Eine responsive Umsetzung demo- 
kratisch gefällter Entscheidungen wird allerdings in dem Maße behindert, in welchem die Regierungsfähigkeit der politischen Entscheidungsträger eingeschränkt wird. Im Rahmen des Demokratiebarometers, in welchem hauptsächlich etablierte Demokratien betrachtet werden, wird diese Einschränkung nicht primär als Behinderung durch nicht legitimierte Akteure oder als mögliche Restriktionen durch supranationale Akteure gedacht, sondern es wird auf mögliche Ressourcen und Implementationsbedingungen in der entsprechenden Demokratie selber rekurriert. ${ }^{5}$

Regierungen benötigen Kontrolle über das politische Geschehen bzw. eine ausreichende Handlungsfähigkeit, um demokratisch gefällte Entscheidungen effizient umsetzen zu können. Eine unzureichende Implementierung infolge mangelnder Ressourcen oder mangelhafter Staatsverwaltung schwächt die Qualität der Demokratie, weil demokratisch getroffene Entscheidungen nicht umgesetzt werden. Eine demokratietheoretische Überlegung, die in nahezu allen Demokratieindizes unberücksichtigt bleibt.

Die Handlungsfähigkeit einer Regierung nimmt ceteris paribus zu, wenn sie über Ressourcen (Komponente 1) verfügt, die eine unparteiische und effektive Umsetzung demokratisch getroffener Entscheidungen ermöglicht. Das bedeutet erstens, dass die gewählte Regierung auf große öffentliche (diffuse und spezifische) Unterstützung zählen kann (Chanley et al. 2000; Rudolph und Evans 2005; Schiller 1999; Tsebelis 1995). Darüber hinaus beeinflusst auch der - durch die Verfassung vorgegebene wie auch effektiv ausgenutzte - Zeithorizont die Regierungsfähigkeit: eine hohe Regierungsstabilität ermöglicht eine kontinuierlichere und damit verlässlichere Umsetzung der Wählerpräferenzen und Erledigung der Staatsaufgaben in der Sicherheits-, Wohlfahrts- und Gerechtigkeitsproduktion, die die Bürger erwarten (Harmel und Robertson 1986).

Die zweite Komponente umfasst Subkomponenten, welche die Bedingungen zur effizienten Implementierung von Policies bzw. zur effizienten Regierungsführung messen. Die Umsetzung von demokratisch getroffenen Regierungsentscheidungen wird erschwert, wenn sie auf Widerstand in Teilen der Bevölkerung stoßen. Dazu können auch Streiks und Demonstrationen gerechnet werden. ${ }^{6}$ In einem viel stärkeren Maße gilt dies für illegitime Umsturzversuche durch Guerillaaktivitäten, gewaltsame Autonomiebestrebungen oder durch die Einflussnahme nicht legitimierter militärischer oder religiöser Akteure. Im Gegensatz dazu kann eine effiziente Verwaltung den Vollzug demokratischer Ent-

5 Wir gehen dabei davon aus, dass Globalisierung im Sinne von Denationalisierung und Internationalisierung der Märkte auf die Qualität von Demokratien einwirkt. Es ist allerdings umstritten, ob diese Einwirkung verhindernden oder aber ermöglichenden Charakter hat (für einen Überblick vgl. Guillén 2003). Mit Hilfe des Demokratiebarometers kann dieser Frage nachgegangen werden; allerdings nur dann, wenn externe Einschränkungen nicht ins Maß einfließen.

6 Hier zeigt sich exemplarisch die Zielinkongruenz einzelner Funktionen. Sowohl Regierungsfähigkeit als auch öffentliche Artikulation werden als wichtige Funktionen einer guten Demokratie betrachtet. Demonstrationen können einerseits die Umsetzung demokratisch getroffener Entscheidungen behindern; sie können andererseits aber ein Indiz für eine aktive Öffentlichkeit sein. Solche Trade-Offs, die der repräsentativen Demokratie inhärent sind (Alonso et al. 2010) auch kenntlich zu machen, ist ebenfalls ein Ziel des Demokratiebarometers. Freilich wird mit dem Ziel der Vermeidung von Redundanz und hier konkret mit dem Ziel der Vermeidung artefaktischer Zusammenhänge - darauf geachtet, dass kein Indikator zur Messung mehrerer Konzepte herangezogen wird. 
scheidungen wesentlich vereinfachen und so mithelfen, Regierungsfähigkeit und OutputLegitimität zu steigern (Scharpf 1999).

\subsubsection{Transparenz}

Fehlende Transparenz hat verschiedene negative Auswirkungen auf die Qualität einer Demokratie: „Secrecy provides the fertile ground on which special interests work; secrecy serves to entrench incumbents, discourage public participation in democratic processes, and undermine the ability of the press to provide an effective check against the abuses of government" (Stiglitz 1999, S. 14). Da fehlende oder mangelhafte Transparenz gewisse Interessengruppen übervorteilt, den breiten, öffentlichen Informationsfluss untergräbt und politische Partizipation hemmen kann, stellt Intransparenz eine Gefahr für das Gebot der politischen Gleichheit in der Demokratie dar. Transparenz bedeutet dabei einerseits Absenz von Geheimhaltung (Komponente 1). Stiglitz (1999) weist darauf hin, dass Geheimhaltung Korruption und Bestechung fördert. Korruption wird in etablierten Demokratien deshalb als Anzeichen für geringe politische Transparenz gedeutet (Lindstedt 2005; Rosendorff 2004). Die ungerechtfertigte Übervorteilung partikulärer Interessen wird insbesondere auch im Zusammenhang mit Parteienfinanzierung diskutiert. Wir argumentieren, dass Regeln, die eine Offenlegung von Einnahmen und Ausgaben von Parteien verlangen, Geheimhaltung einschränken und Transparenz fördern.

Andererseits sollte eine Demokratie insgesamt über gute Voraussetzungen für einen transparenten politischen Prozess verfügen. Partizipation, öffentliche Artikulation, Wettbewerb und Kontrolle sind abhängig davon, wie einfach Informationen über den politischen Prozess erhältlich sind und wie groß seitens der Regierenden die Bereitschaft zur offenen Kommunikation ist. Stiglitz (1999) spricht in diesem Zusammenhang von einer Kultur der Offenheit. Allerdings kann schon auf der Verfassungsebene eine wirksame Informationsfreiheitsgesetzgebung vorschreiben, dass Informationen zu politischen Entscheidungen leicht zugänglich sind (Islam 2006). Von zentraler Bedeutung für die Transparenz ist, wie offen über Regierungspolitik berichtet wird: Medien, die über politische Entscheidprozesse berichten, dürfen keinem politischen Druck oder Zensur ausgesetzt werden. Zudem sollte die Medienregulierung eines Landes weder Medieninhalte noch die Handlungsfähigkeit von Medienunternehmen einschränken. Transparenz ist eine wichtige Funktion, die eine gleichberechtigte Kontrolle der Regierenden durch die Regierten erst wirklich effektiv macht.

\subsubsection{Partizipation}

Partizipationsrechte und -möglichkeiten sollten in einer guten Demokratie nicht nur gleich verteilt sein, sondern auch möglichst gleichmäßig und umfassend genutzt werden (Pateman 1970; Barber 1984; Teorell 2006). Entsprechend steht erstens die Gleichheit der Partizipation als eine Komponente im Zentrum dieser Funktion. Weil es unter der Annahme normativer demokratischer Gleichheit keine unabhängige Kriterien für den Ausschluss bestimmter Individuen von politischen Entscheidungen geben kann, wird argumentiert, dass alle Individuen, die von einer politischen Entscheidung betroffen sind, auch die Möglichkeit haben müssen, an dieser Entscheidung teilzuhaben (Kel- 
sen 1925; Dahl 1998). Dies wird i.d. R. durch Partizipationsrechte bestimmt (Blais et al. 2001; Paxton et al. 2003). Daneben sollte sich kein Individuum aufgrund gewisser sozialer Charakteristika oder mangels Ressourcen daran hindern lassen, am politischen Prozess teilzunehmen - sei dies im Rahmen von Wahlen oder alternativen Partizipationsformen (Demonstrationen und Petitionen). Ungleiche Partizipation führt dazu, dass nicht alle Interessen im politischen System vertreten sind und somit repräsentativ-responsives Handeln seitens der politischen Repräsentanten eingeschränkt bleibt (Alonso et al. 2010; Lijphart 1997; Rueschemeyer 2004; Teorell et al. 2007).

Zweitens ist gerade die effektive Partizipation entscheidend, denn eine hohe politische Beteiligung geht i.d.R. Hand in Hand mit sozial gleichmäßiger, d.h. möglichst geringer gesellschaftlicher Selektivität bei der politischen Partizipation (Lijphart 1997). Auch bei dieser Komponente werden verschiedene Partizipationsmöglichkeiten und -formen berücksichtigt, da die verschiedenen Beteiligungsopportunitäten nicht überall die gleiche Bedeutung haben. Entsprechend wird die Höhe der Partizipation bei konventionellen (Legislativ- und Exekutivwahlen sowie auch Referenden) und unkonventionellen Beteiligungsformen (Demonstrationen und Petitionen) gemessen. Auch die effektive Partizipation kann schließlich durch formelle Mechanismen gefördert werden: Eine Vereinfachung der Partizipation etwa durch flexible Wahlorte oder Möglichkeiten zur frühzeitigen Stimmabgabe können die allgemeine Partizipationsbereitschaft erhöhen. Die Beteiligung wird hingegen in jenen Ländern eingeschränkt, bei denen eine Registrierungspflicht vorgesehen ist.

\subsubsection{Repräsentation}

Repräsentation bedeutet, dass möglichst alle Individuen innerhalb einer politischen Einheit politische Mitsprache genießen und im politischen System vertreten sein sollten. Responsive, repräsentative Demokratien müssen gewährleisten, dass möglichst alle gesellschaftlichen Interessen gleichgewichtig in gewählten Gremien vertreten werden. Deshalb ist einerseits eine adäquate deskriptive Repräsentation von Minderheiten im Parlament ein entscheidendes Kriterium (Mansbridge 1999; Wolbrecht und Campbell 2007). Substantielle Repräsentation fokussiert andererseits auf eine adäquate Inklusion von Präferenzen (Mansbridge 2003). Sie wird mit der ersten Komponente bestimmt, welche zwei Subkomponenten umfasst. Die erste misst den Grad an Disproportionalität zwischen Stimm- und Sitzanteilen sowie die Kongruenz zwischen der Verteilung von Links-RechtsPräferenzen in der Bevölkerung und dem Parlament. Schlechte Übereinstimmungen von Wählerstimmen- und Sitzanteilen oder Inkongruenzen von Wähler- und Parteipräferenzen sind Zeichen einer ungleichen Einbindung von individuellen Präferenzen (Holden 2006; Urbinati 2010; Urbinati und Warren 2008). Die Inklusion von Präferenzen kann durch strukturelle Opportunitäten beeinflusst werden: so zeigen verschiedene empirische Beiträge (für einen Überblick vgl. Powell 2004), dass eine hohe relative Sitzzahl substantielle Repräsentation begünstigt. Darüber hinaus bieten direktdemokratische Verfahren eine unmittelbare Möglichkeit zur Inklusion von Präferenzen.

Die zweite Komponente besteht aus drei Subkomponenten, welche die deskriptive Repräsentation von Minderheiten bestimmen. Als Minderheiten werden dabei einerseits ethnische Minderheiten verstanden, deren Zugang zu Machtpositionen gemessen 
wird (Alonso 2010; Banducci et al. 2004). Andererseits gelten Frauen als strukturelle Minderheiten, deren adäquate Vertretung erstens eine zentrale Forderung deskriptiver Repräsentationsansätze darstellt und zweitens auch hundert Jahre nach Einführung des Frauenwahlrechts nirgends vollständig eingelöst wird (Dahlerup 2010). Um GenderGleichgewichte zu messen, werden effektive Vertretungsquoten und spezifische verfasste politische Rechte für Frauen jeweils zu Subkomponenten verdichtet.

\section{Demokratiequalität messen}

Auf der Basis unseres differenzierten theoretischen Demokratiekonzepts mittlerer Reichweite kann nun der Grad an Demokratiequalität in einem Land für einen gegebenen Zeitpunkt gemessen werden. Dazu werden zuerst anhand einer Reihe von Indikatoren die Komponenten der verschiedenen Funktionen gemessen, um so den Erfüllungsgrad jeder einzelnen Funktion zu bestimmen. Mittels Aggregierung der entsprechenden Funktionen und danach der Prinzipien zum Konzept ,Demokratiequalität' erhalten wir dann ein Maß, mit welchem die Güte der jeweiligen gesamten Demokratie erfasst und im Längs- und Querschnitt verglichen werden kann.

Freilich basieren die einzelnen Messschritte auf wichtigen Prämissen. Um die Kritik an aktuellen Demokratiemessungen zu berücksichtigen, sollen diese Prämissen theoretisch untermauert, transparent und intersubjektiv nachvollziehbar dargestellt werden. Drei zentrale Herausforderungen stellen sich beim eigentlichen Messvorgang (Müller und Pickel 2007; Munck und Verkuilen 2002). Erstens muss festgelegt werden, welche Eigenschaften Indikatoren aufweisen müssen, damit sie sich für die Bestimmung der einzelnen Komponenten bzw. Subkomponenten eignen (Abschn.3.1). Zweitens müssen geeignete Ober- und Untergrenzen der Messung festgelegt werden (Abschn. 3.2). Drittens ist entscheidend, auf welche Weise die einzelnen Indikatoren zu Komponenten, Funktionen und dem Indikator für Demokratiequalität verdichtet werden (Abschn. 3.3).

\subsection{Geeignete Indikatoren}

Wie oben diskutiert, werden die drei Prinzipien in neun Funktionen der Demokratie ausdifferenziert, die durch jeweils zwei Komponenten näher bestimmt werden. Von diesen werden wiederum mehrere Subkomponenten deduziert, welche schließlich mittels verschiedener Indikatoren gemessen werden. Insgesamt bestehen die neun Funktionen bzw. 18 Komponenten aus 51 Subkomponenten und 100 Indikatoren. $^{7}$

7 Es werden hier nicht alle Indikatoren einzeln besprochen. Ein erster Überblick über die in diesem Beitrag verwendeten Indikatoren findet sich im Anhang. Eine detaillierte Demonstration und Diskussion aller Funktionen (Konzeptbäume), die Quellen und Ausprägungen aller Indikatoren, ausführliche Hinweise zur Messmethode sowie die Rohdaten für alle Indikatoren, Komponenten und Funktionen sowie der Index ,Demokratiequalität' lassen sich unter www. democracybarometer.org abrufen. 
Teilweise handelt es sich bei den Indikatoren um eigene Erhebungen oder Auswertungen von Quellenmaterial. Die meisten der 100 Indikatoren wurden jedoch aus einer grossen Sammlung von mehr als 300 Sekundärdaten-Indikatoren anhand von spezifischen Kriterien ausgewählt. Bei der Selektion der Indikatoren haben wir versucht die Mängel der bisherigen Demokratieindizes zu berücksichtigen.

1. Es wurde versucht, Indikatoren zu vermeiden, die auf Expertenbefragen beruhen, da deren Reliabilität z. T. fragwürdig und die Kategorienzuordnung zumeist hochgradig intransparent ist (Bollen und Paxton 1998, 2000). Anstelle von Einschätzungen durch sogenannte Länderexperten verwendet das Demokratiebarometer möglichst Indikatoren, die auf ,objektiven“ Daten aus statistischen Sekundärquellen oder repräsentativen Bevölkerungsumfragen beruhen.

2. Ein Hauptziel des Demokratiebarometers ist es, die effektive Demokratiequalität zu messen. Mit anderen Worten sollen ,institutionalistische Fehlschlüsse“ (Abromeit 2004) möglichst vermieden werden. Deshalb fokussieren wir nicht nur auf die Existenz von Institutionen wie dies etwa beim Polity-Index der Fall ist, sondern beziehen insbesondere auch die „Verfassungswirklichkeit“ (Meyer 2005) oder die effektive Wirkung von Institutionen mit ein. In jeder Funktion findet sich deshalb jeweils mindestens eine Subkomponente, deren Indikatoren ,rules in law` erfassen als auch mindestens eine Subkomponente, deren konstituierende Indikatoren ,rules in use messen. Damit sollen die demokratischen Normen des Verfassungsbuchstaben und die Manifestation in der Verfassungswirklichkeit erfasst werden. Würden nur erstere gemessen, wäre noch nicht viel über deren Umsetzung gesagt. Würde nur letztere berücksichtigt, könnten wir nicht das Spektrum demokratischer Möglichkeiten erfassen, die, selbst dann, wenn sie nicht umfangreich genutzt werden, doch ein Element der Demokratiequalität darstellen, da sie die Möglichkeitsstruktur der Demokratie in den Blick nehmen.

3. Um Messfehler zu reduzieren wurde versucht, zur Messung jeder Subkomponente mindestens zwei Indikatoren aus unterschiedlichen Quellen heranzuziehen (Bollen 1993; Kaufmann und Kraay 2008).

\subsection{Skalierung}

Eine der am häufigsten missachteten Herausforderung in der empirischen Demokratiemessung ist die theoretische Fundierung der Skalierung der verwendeten Indikatoren (Munck und Verkuilen 2002). Von großer Bedeutung ist dabei die Bestimmung von Unter- und Obergrenzen bzw. die Skalierung der Indikatoren nach bestimmten Regeln. Grundsätzlich sind drei solche Regeln denkbar:

1. Wir könnten unsere Indikatoren an theoretisch deduzierten Bezugswerten ausrichten. In diesem Fall müsste uns die Theorie mit Minima und Maxima für jeden Indikator aushelfen. Während dies für formale Institutionen wahrscheinlich relativ einfach wäre, dürfte es sich bei vielen Indikatoren hingegen als nahezu unmöglich heraus- 
stellen, Schwellenwerte für tatsächliche Wirkungen von Institutionen zu bestimmen. ${ }^{8}$ Darüber hinaus wäre die Definition von theoretischen Minima und Maxima insofern bedenklich, als dass, wie bereits eingangs erwähnt, keine universelle Theorie von Demokratie existiert. Die Definition der Schwellenwerte wäre abhängig von der normativen Ausgangsposition und würde deshalb sehr unterschiedlich ausfallen. Für einen minimalistischen Demokratieentwurf wäre etwa eine Wahlbeteiligung von $50 \%$ unproblematisch. Konsequenterweise wäre 100\% Wahlbeteiligung nicht notwendigerweise ein theoretisches Maximum. Anhänger der partizipatorischen Demokratietheorie würden jedoch nicht nur universelles Wahlrecht, sondern auch eine möglichst hohe Wahlbeteiligung fordern. Darüber hinaus lassen sich für verschiedene Indikatoren überhaupt keine theoretischen Maxima oder Minima finden.

2. Ober- und Untergrenzen könnten durch internationale, ,neutrale" Standards für Demokratiequalität vorgegeben werden. Allerdings lassen sich solche Standards nur für die wenigsten unserer Indikatoren finden. ${ }^{9}$

3. Wir optieren deshalb für die dritte Möglichkeit, welche die Indikatoren nach ,best ${ }^{\star}$ bzw. ,worst practice'-Verfahren klassiert. Dazu wurde folgendes Vorgehen gewählt. Zuerst wurde auf der Basis etablierter Demokratieindizes ein Ländersample erstellt, welches für den Zeitraum zwischen 1995 und 2005 alle etablierten Demokratien mit mehr als 250.000 Einwohnern enthält. ${ }^{10}$ Aufgrund dieses Samples, welches quasi das ,Universum aller etablierter Demokratien' abbildet, wird die Skalierung vorgenommen sowie die Beziehungsstruktur zwischen den Indikatoren einer Subkomponente festgelegt, um beides dann später als eine Art ,blue print' auf weitere Länder und

8 Zwei Beispiele sollen als Illustration dienen: Wie groß ist die optimale Anzahl von Parteien? Wie viele Streiks pro Jahr kennzeichnen eine minimale Demokratiequalität? Es könnte argumentiert werden, dass diese Schwierigkeit für die Konzentration auf formale Institutionen sprechen würde. Mit unserem Demokratiekonzept schlagen wir hingegen vor, dass Demokratiequalität umfassender gemessen werden muss. Freilich lässt sich das Demokratiebarometer auch auf Indikatoren reduzieren, die lediglich Institutionen messen. Es steht den Anwender/ Innen unseres Instruments frei, einer institutionalistischen Minimaldefinition der Demokratie zu folgen und sich die entsprechenden Indikatoren auszuwählen. Die Transparenz der Daten macht eine solche Vorgehensweise möglich.

9 Ein Beispiel für entsprechende Standards wäre etwa die UNO-Menschenrechtscharta (z. B. Humana 1992).

10 Ein Land gilt dann als etablierte Demokratie, wenn es in allen elf Untersuchungsjahren (19952005) bei Freedom House mindestens den Wert 1,5 und bei Polity mindestens den Wert 8,0 aufweist, also über einen längeren Zeitraum hinweg konstant als etablierte Demokratie eingestuft wird. Folgende 34 Länder erfüllen diese Kriterien: Australien, Bahamas, Barbados, Belgien, Costa Rica, Dänemark, Deutschland, Finnland, Frankreich, Großbritannien, Island, Irland, Italien, Japan, Kanada, die Kapverdischen Inseln, Luxemburg, Malta, Mauritius, Neuseeland, die Niederlande, Norwegen, Österreich, Polen, Portugal, Schweden, Slowenien, Südafrika, Spanien, die Schweiz, die Tschechische Republik, Ungarn, die USA und Zypern. Aufgrund zu vieler fehlender Daten mussten Bahamas, Barbados, die Kapverdischen Inseln und Mauritius ausgeschlossen werden. Unser , blue print ${ }^{6}$-Sample umfasst also 30 Länder und elf Jahre (330 Länderjahre). 
Jahre im Demokratiebarometer anzuwenden. ${ }^{11}$ Für die eigentliche Skalierung wurden innerhalb dieses ,blue print'-Samples (30 Länder und elf Jahre, also total 330 Länderjahre) alle Indikatoren so standardisiert, dass für jeden Indikator die höchste (im Sinne des Erfüllungsgrads einer Funktion) Ausprägung mit dem Wert 100 und die niedrigste Ausprägung mit dem Wert 0 versehen wurde. ${ }^{12}$ Neben den Einwänden, die oben bereits gegen die alternativen Skalierungsverfahren vorgebracht wurden, gibt es mindestens drei weitere Gründe für die Wahl des ,best practice'-Vorgehens:

- Wir definieren Demokratie als eine politische Ordnung, die sich aufgrund kontinuierlicher politischer und gesellschaftlicher Aushandlungsprozesse beständig neu bestimmt und verändert. Wir gehen konsequenterweise davon aus, dass jede Demokratie den neun Funktionen und drei Prinzipien infolge historischer Pfade und normativer Präferenzen unterschiedliche Wichtigkeit zuschreibt. Was wir also messen sollten, ist das momentan existierende und justierbare empirische Maximum; mit anderen Worten: die ,best practice‘ für jede Funktion. Diese lässt sich zudem jederzeit neu anpassen. ${ }^{13}$ Das ist im Vergleich zu anderen Demokratiemaßen ein nicht zu unterschätzender Vorteil bei Längsschnitt-Analysen. Während Verbesserungen über die Zeit mit einem einmal definierten und fixen Maximum nicht mehr adäquat erfasst werden können, erlaubt die ,best practice'-Messung eine flexible Anpassung nach oben und nach unten.

- Ein wichtiges Ziel des Projekts ist der Vergleich verschiedener etablierter Demokratien. Die Bestimmung von ,best practice‘ kommt diesem Anliegen in adäquater Weise entgegen (vgl. dazu auch Beetham 2004), denn sie erlaubt die Darstellung von Zielkonflikten zwischen verschiedenen Prinzipien bzw. Funktionen, von möglichen Trade-Offs und von verschiedenen realen Erscheinungsformen von Demokratien (siehe nachfolgend).

- Was die Mindestschwelle betrifft, ist hervorzuheben, dass es nicht Ziel des Demokratiebarometers ist, zu definieren, ob ein Land eine Demokratie ist oder nicht. Wir wollen vielmehr den Gehalt der Demokratie von bereits etablierten Demokratien bestimmen. Werte von 0 oder Minuswerte sind deshalb möglich.

11 Daten für rund 45 weitere Länder und den Zeitraum 1990-2007 werden z.Z. erhoben und gemäß dem ,blue print'-Sample rekodiert.

12 Werden weitere Länder oder Jahre mit einbezogen, ist es also möglich, dass sich Werte unter 0 oder über 100 ergeben. 0 bedeutet also nicht ,keine Demokratie', sondern 0 (oder Werte unter 0 ) bedeutet, dass ein Land bei einem Indikator schlechter abschneidet als das schlechteste Land (bei diesem Indikator) der ,blue print'-Länder zwischen 1995 und 2005.

13 Im Prinzip braucht es keine Anpassung, da die Skala nach oben und unten offen ist. Werte über 100 bedeuten, dass ein Land bei einem Indikator einen höheren Wert erreicht, als dies im ,blue print'-Sample (30 Länder in elf Jahren) erreicht wurde. Der Demokratiebarometer ist zudem mit neuen Indikatoren erweiterbar, wenn sie die Bedingungen, wie sie unter Abschn.3.1 formuliert wurden, erfüllen. Wir laden die Forschergemeinschaft ein, die Indikatorenliste zu erweitern und so die Messung von Demokratiequalität weiter zu verbessern (vgl. dazu auch www.democracybarometer.org). 


\subsection{Aggregierung}

Die dritte Herausforderung, der jede Indexbildung begegnen muss, ist die Frage der Aggregierung: wie sollen die Indikatoren zu Subkomponenten, Komponenten, Funktionen, Prinzipien und schließlich zum Index ,Demokratiequalität" verdichtet werden? Damit verbunden ist zudem die Frage der Gewichtung der einzelnen Bestandteile. Wir haben uns -nach ausführlichen empirischen Tests - für die nachfolgend beschriebene Vorgehensweise entschieden.

Um Demokratiequalität zu messen, gehen wir den in der theoretischen Deduktion gefolgten Weg in umgekehrter Richtung zurück - von konkreten Indikatoren zum abstrakten Konzept. Zuerst werden die ausgewählten Indikatoren gemäß ,best practice‘ skaliert. Der arithmetische Mittelwert der Indikatoren bildet dann den Wert der entsprechenden Subkomponente. Der , einfache ' Mittelwert spiegelt die Überlegung wieder, dass die Indikatoren einer Subkomponente das gleiche Konzept messen müssen und entsprechend kompensierbar sein sollten. Auf die gleiche Weise werden im nächsten Schritt aus den Subkomponenten die Komponenten gebildet.

In den nachfolgenden Aggregationsschritten (von Komponenten zu Funktionen; von Funktionen zu Prinzipien; von Prinzipien zum Gesamtaggregat ,Demokratiequalität‘) fließen zwei Ideen ein, die bereits im theoretischen Teil erörtert wurden: wir gehen erstens davon aus, dass es sich bei den Komponenten, Funktionen und Prinzipien jeweils um notwendige Bedingungen für die jeweils nächste Aggregatsstufe handelt. Zweitens nehmen wir an, dass es Demokratien unterschiedlich gut gelingt, eine optimale Balance zwischen den Komponenten, bzw. den Funktionen und den Prinzipien herzustellen. Wir errechnen deshalb den Wert der jeweiligen höheren Stufe mit Hilfe einer Formel, welche erstens die Idee der notwendigen Bedingungen aufnimmt und zweitens nicht nur hohe Werte belohnt, sondern auch Inkongruenzen zwischen Wertepaaren bestraft. ${ }^{14}$

14 Die Aggregationsformel soll nicht nur Progression abbilden (vermittels Multiplikation) und ungleiche Betonung unterschiedlicher Elemente (Komponenten, Funktionen, Prinzipien) adäquat (d.h. mit zunehmendem Ungleichgewicht zunehmend) bestrafen, sondern sie soll auch abnehmenden Grenznutzen bei zunehmender Demokratiequalität abbilden. Wir haben uns deshalb für eine Arkustangens-Funktion entschieden:

$$
\text { Funktionswert }=(\arctan (\text { Komponente } 1 * \text { Komponente } 2) * 1.2 / 4000) * 80 .
$$

Bei drei Elementen, d.h. bei der Aggregierung der Funktionen zu Prinzipien bzw. der Prinzipien zu Demokratiequalität wird die gemittelte Summe der paarweisen Funktionenwerte herangezogen:

$$
\begin{aligned}
\text { Prinzipwert }= & \{[(\arctan (\text { Komponente } 1 * \text { Komponente } 2) * 1.2 / 4000) * 80] \\
& +[(\arctan (\text { Komponente } 1 * \text { Komponente } 3) * 1.2 / 4000) * 80] \\
& +[(\arctan (\text { Komponente } 2 * \text { Komponente } 3) * 1.2 / 4000) * 80]\} / 3 .
\end{aligned}
$$

Komplexer wird die Formel, wenn die Werte einzelner Elemente unter den Nullbereich fallen. Dies ist im hier behandelten Blueprint-Sample natürlich nicht der Fall, wird aber in erweiterten Datensammlungen möglich sein. Eine ausführliche Diskussion zu diesen Fällen sowie zur hier verwendeten Formel findet sich im Methodenhandbuch unter www.democracybarometer.org. 
Unser Vorgehen kann (und soll) natürlich kritisiert werden. So könnte etwa aus einer liberalen Sichtweise heraus argumentiert werden, dass die Funktionen ,Rechtsstaatlichkeit', ,individuelle Freiheiten' und ,Wettbewerb' stärker gewichtet werden müssten als die restlichen Funktionen. Aus einer rein partizipatorischen Perspektive müsste der Funktion Partizipation ein besonderes Gewicht beigemessen werden. Auf der Basis unseres ausgewiesenen Konzeptes mittlerer Reichweite und der Interdependenz aller Funktionen gebietet die Logik, alle Funktionen gleich zu gewichten. Nichtsdestotrotz sind andere Gewichtungen möglich. Allerdings gilt es dann, die theoretische Pflicht zu respektieren und den genauen Gewichtungsfaktor jenseits des Arbiträren zu begründen. ${ }^{15}$

\section{Die Qualität von Demokratien - erste empirische Resultate}

Im Folgenden werden erste vorläufige Resultate präsentiert und ein Einblick in die Analysemöglichkeiten gewährt, die mit dem Demokratiebarometer einhergehen. Dies soll gleichzeitig die Plausibilität des Messinstrumentes aufzeigen.

Wir argumentieren, dass der Erfüllungsgrad der Funktionen innerhalb eines etablierten demokratischen Systems das Ergebnis politischer und gesellschaftlicher Aushandlungsprozesse darstellt. Die unterschiedliche Balance der neun Funktionen lässt sich anhand von Netzdiagrammen darstellen. In Abschn.4.1. präsentieren wir deshalb zuerst eine deskriptive Analyse unterschiedlicher Formen von Demokratie. Das Demokratiebarometer eignet sich des Weiteren auch für Längs- und Querschnittvergleiche der Demokratiequalität insgesamt oder einzelner Funktionen (Abschn. 4.2).

\subsection{Formen von Demokratien - deskriptive Analysen}

In einer ersten, etwas naiven Herangehensweise könnte vermutet werden, dass ein demokratisches Regime, das bei allen Funktionen den höchsten Erfüllungsgrad aufweist, auch die höchste Demokratiequalität erreicht. Unter der Annahme, dass die neun Funktionen in einem Spannungsverhältnis stehen, scheint eine simultane Maximierung aller neun Funktionen allerdings sowohl aus theoretischer wie auch aus empirischer Warte unwahrscheinlich. Wir gehen deshalb davon aus, dass verschiedene empirische Realisierungen von Demokratien unterschiedliche Optima bzgl. der Kombination der neun Funktionen finden. Diese unterschiedlichen Optima lassen sich anhand von Netzdiagrammen darstellen, bei denen die einzelnen Achsen die neun Funktionen repräsentieren. Zur Illustration werden in Abb. 2 die Netzdiagramme der drei Länder für jeweils drei Jahre abgebildet, die bereits in der Einleitung Erwähnung fanden: Finnland, Italien und die USA.

Wie eingangs erwähnt, finden sich bei etablierten Demokratieindizes wie Freedom House oder Polity zwischen diesen Ländern keine Unterschiede. Die Netzdiagramme zeigen jedoch eine recht deutliche Varianz hinsichtlich der Form und der Größe der Netzflächen zwischen den Ländern. Anhand der Formen lassen sich zudem unterschiedliche

15 Wir laden die Forschergemeinschaft explizit dazu ein, mit den Daten des Demokratiebarometers andere Gewichtungs- und Aggregierungsverfahren zu testen und anzuwenden. 


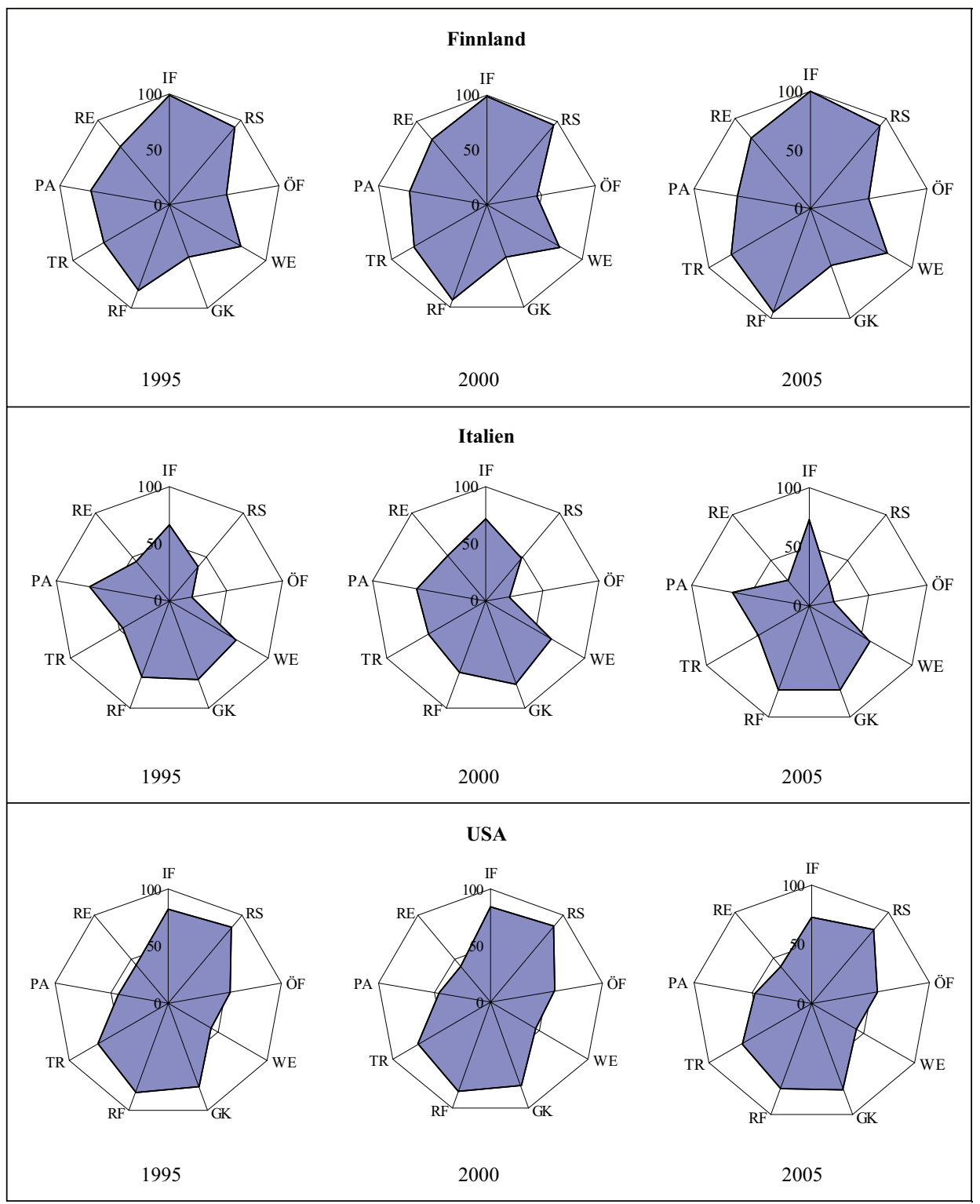

Abb. 2: Demokratiebarometer - Netzdiagramme. (IF Individuelle Freiheiten, $R S$ Rechtsstaatlichkeit, $\ddot{O F}$ Öffentlichkeit, $W E$ Wettbewerb, $G K$ Gewaltenkontrolle, $R F$ Regierungs- und Implementierungsfähigkeit, $T R$ Transparenz, $P A$ Partizipation, RE Repräsentation) 
Realisierungen von Demokratie beobachten: in den drei Ländern werden verschiedene Funktionen unterschiedlich und in unterschiedlichem Grad erfüllt.

Darüber hinaus können auch Veränderungen über die Zeit in den Ländern selbst festgestellt werden. Die Interpretation all dieser Unterschiede soll hier nicht Gegenstand sein. Es kann aber festgehalten werden, dass sich mit dem Demokratiebarometer verschiedene Vergleichsmöglichkeiten eröffnen, welche sich zudem anschaulich darstellen lassen.

\subsection{Demokratiequalität im Vergleich}

Auch wenn die neun Funktionen kaum gleichzeitig maximiert werden können, so kann ihr Zusammenspiel doch derart optimiert werden, dass sich die gesamte Demokratiequalität erhöht. Wir gehen also davon aus, dass sich Demokratien hinsichtlich der von uns bestimmten Demokratiequalität (Gesamtaggregat) unterscheiden und dass sich die Qualität einer Demokratie über die Zeit verändert. Hinsichtlich unseres Gesamtindex für Demokratiequalität lassen sich für die 30 , blue print'-Länder für die Jahre 1995-2005 folgende Beobachtungen machen.

Im Zeitvergleich zeigt sich im Schnitt über alle 30 Demokratien insgesamt eine leichte Zunahme an Demokratiequalität von 1995 bis 2005. Die mittlere Demokratiequalität aller 30 Staaten liegt 1995 bei 63,1. Sie steigt bis 2000 auf 66,6 an und nimmt dann bis 2005 wieder auf 65,5 ab. ${ }^{16}$ Von einer häufig behaupteten kontinuierlich zunehmenden Krise der Demokratie kann also nicht gesprochen werden (Crozier et al. 1975; Pharr und Putnam 2000), da bereits das Ausgangsniveau 1995 relativ hoch ist und sich von da weiterentwickelt. ${ }^{17}$ Freilich variieren die einzelnen Länder relativ stark. Werden lediglich die Differenzen der Länderwerte zwischen 2005 und 1995 betrachtet, können grob drei Ländergruppen unterschieden werden.

Insgesamt verschlechtert sich die Demokratiequalität zwischen 1995 und 2005 in neun Ländern: in Italien (Rückgang von 2005 bis 1995 um - 9,3 DQ-Punkte), in der Tschechischen Republik $(-7,2)$, Portugal $(-5,0)$, in den USA $(-2,2)$, in Costa Rica $(-1,8)$, in Irland $(-1,4)$, in Australien $(-1,3)$ in Frankreich $(-1,0)$, und in Deutschland $(-1,0)$. In den restlichen 21 Ländern zeigt sich eine Verbesserung der Demokratiequalität über die Zeit. Diese ist in 14 Ländern unterdurchschnittlich ausgeprägt (unter 4,9), nämlich in Dänemark $(0,9)$, Ungarn $(1,2)$, Finnland $(1,8)$, Norwegen $(2,4)$, Zypern $(2,5)$, Luxemburg $(2,5)$, Schweden $(2,6)$, Spanien $(2,8)$, Österreich $(2,9)$, Slowenien $(2,9)$, Belgien $(3,2)$, den Niederlanden $(3,9)$, Neuseeland $(3,9)$ und Südafrika $(4,1)$. In den restlichen sieben Ländern zeigt sich eine überdurchschnittliche Zunahme der Demokratiequalität (Zunahme von mehr als 4,9 zwischen 1995 und 2005): in Kanada (5,8), Island (6,3), Polen $(7,8)$, Grossbritannien $(10,0)$, Malta $(11,3)$, Japan $(11,8)$ und in der Schweiz $(12,8)$.

Die Veränderungen in der Demokratiequalität über die Zeit schlagen sich teilweise auch in den Rangfolgen der Länder hinsichtlich ihrer demokratischen Qualität nieder (Tab. 1).

16 Den geringsten Wert in den 330 Länderjahren weist Costa Rica im Jahr $2005(29,2)$ und den höchsten Wert weist Dänemark in den Jahren 2003 und 2004 auf $(88,7)$.

17 Es kann vermutet werden, dass sich der Knick in den Jahren 1999/2000 mindestens teilweise auf die dotcom-Krise zurückführen lässt. 
Tab. 1: Die Qualität etablierter Demokratien (Rangliste für 1995, 1998, 2000, 2003 und 2005; Länder geordnet entsprechend ihres Rangs 2005)

\begin{tabular}{|c|c|c|c|c|c|}
\hline Land & 1995 & 1998 & 2000 & 2002 & 2005 \\
\hline Dänemark & 1 & 1 & 2 & 1 & 1 \\
\hline Finnland & 2 & 2 & 1 & 2 & 2 \\
\hline Belgien & 3 & 5 & 3 & 3 & 3 \\
\hline Island & 6 & 4 & 4 & 4 & 4 \\
\hline Schweden & 5 & 3 & 5 & 5 & 5 \\
\hline Norwegen & 4 & 6 & 6 & 6 & 6 \\
\hline Kanada & 8 & 7 & 7 & 8 & 7 \\
\hline Niederlande & 7 & 8 & 8 & 7 & 8 \\
\hline Schweiz & 19 & 21 & 13 & 12 & 9 \\
\hline USA & 9 & 10 & 10 & 11 & 10 \\
\hline Neuseeland & 14 & 11 & 11 & 13 & 11 \\
\hline Luxemburg & 11 & 9 & 9 & 9 & 12 \\
\hline Deutschland & 10 & 12 & 12 & 10 & 13 \\
\hline Slowenien & 12 & 13 & 15 & 14 & 14 \\
\hline Irland & 13 & 14 & 14 & 16 & 15 \\
\hline Spanien & 17 & 16 & 18 & 15 & 16 \\
\hline Österreich & 18 & 20 & 21 & 18 & 17 \\
\hline Australien & 16 & 17 & 19 & 17 & 18 \\
\hline Portugal & 15 & 15 & 17 & 19 & 19 \\
\hline Ungarn & 20 & 19 & 16 & 20 & 20 \\
\hline Malta & 24 & 24 & 24 & 21 & 21 \\
\hline Zypern & 23 & 23 & 23 & 22 & 22 \\
\hline Tschechische Republik & 21 & 22 & 22 & 24 & 23 \\
\hline Japan & 26 & 29 & 27 & 25 & 24 \\
\hline Grossbritannien & 28 & 26 & 26 & 26 & 25 \\
\hline Italien & 22 & 18 & 20 & 23 & 26 \\
\hline Polen & 27 & 27 & 29 & 27 & 27 \\
\hline Frankreich & 25 & 25 & 25 & 29 & 28 \\
\hline Südafrika & 29 & 28 & 28 & 28 & 29 \\
\hline Costa Rica & 30 & 30 & 30 & 30 & 30 \\
\hline
\end{tabular}

So verschlechtern sich etwa Deutschland und Frankreich innerhalb der Untersuchungsjahre um drei Ränge und Italien und Portugal sogar um vier Ränge, während sich umgekehrt Neuseeland, Malta und Grossbritannien um 3 und die Schweiz gar um 10 Ränge verbessern. ${ }^{18}$

18 Zumindest teilweise lassen sich diese Verbesserungen, die hinsichtlich der Rangpunkte - nicht aber hinsichtlich der effektiven Werte - recht hoch ausfallen, durch institutionelle Reformen erklären: in Neuseeland scheint die Wahlrechtsreform 1994 bzw. die darauf folgenden Wahlen 1996 zu Verbesserungen in der demokratischen Qualität zu führen, während der Rangsprung der Schweiz zwischen 1998 und 1999 unter anderem auf die Totalrevision der Verfassung zurückführbar sein dürfte. 


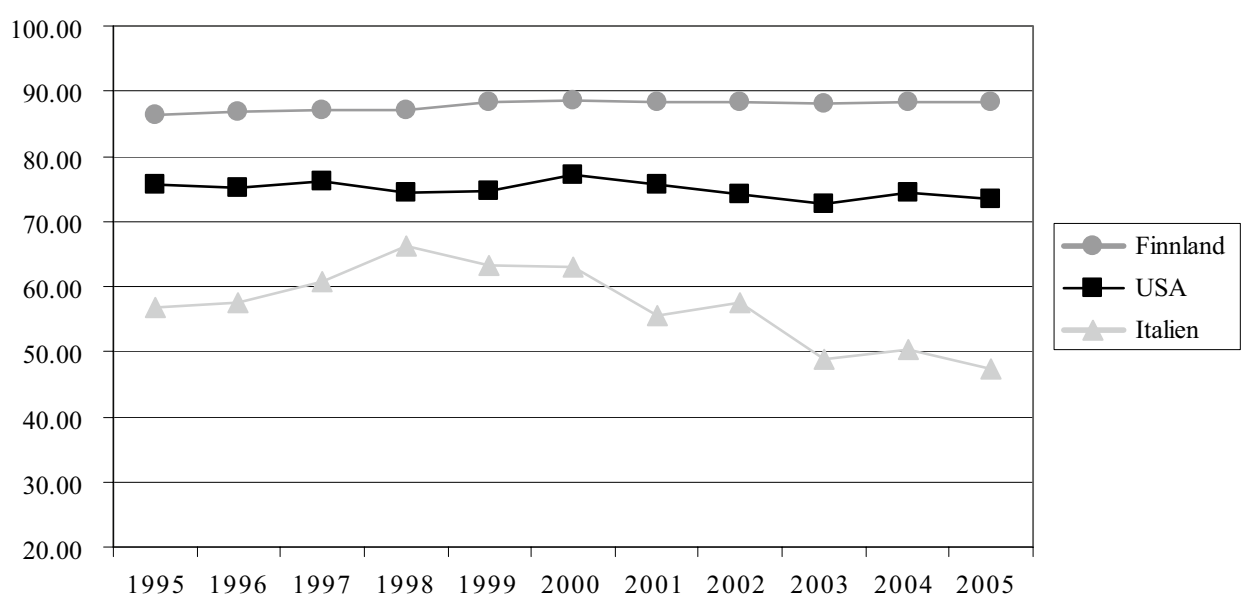

Abb. 3: Entwicklung der Demokratiequalität in Finnland, Italien und den USA

Die Veränderungen über die Zeit lassen sich mit dem Demokratiebarometer noch differenzierter nachzeichnen. Wiederum sollen die drei Beispielländer Finnland, Italien und die Vereinigten Staaten zur Illustration herangezogen werden. Abbildung 3 zeigt, dass Finnland hinsichtlich der allgemeinen Demokratiequalität insgesamt auf einem höheren Niveau liegt als die USA und Italien. Die Entwicklungen in der Demokratiequalität scheinen zudem - zumindest in Italien und den USA - mit Regierungswechseln einherzugehen. Dies kann zumindest als ein Indiz für eine valide Messung betrachtet werden, insbesondere wenn bedacht wird, dass keiner der Indikatoren politische Faktoren wie bspw. die parteiliche Zusammensetzung der Regierung o. ä. erfasst. Natürlich müssen und können hier feinere Untersuchungen erfolgen, um das Potenzial des Demokratiebarometers noch besser auszuschöpfen.

\section{Die Qualität der Qualitätsmessung}

Nachfolgend soll evaluiert werden, ob das Demokratiebarometer ein zuverlässiges Maß ist oder nicht. In der Folge werden deshalb Validitätstests durchgeführt. Es wird dabei zwischen Konstrukt- (Abschn. 5.1) und Kriteriumsvalidität (Abschn. 5.2) unterschieden. Konstruktvalidität bedeutet, dass sich unsere theoretischen Erwartungen hinsichtlich der Funktionen und Komponenten auch empirisch nachzeichnen lassen müssen. Kriteriumsvalidität kann attestiert werden, wenn unser Demokratiemaß mit anderen Faktoren so korreliert, wie es gemäss einschlägigen Theorien erwartet werden kann. Darüber hinaus führen wir endogene Validitätstests durch und gehen dabei den in der empirischen Demokratieforschung am häufigsten gewählten Weg, nämlich den Vergleich unseres Maßes mit bestehenden Indizes, die Demokratie ähnlich messen (Abschn. 5.3). 
Tab. 2: Unterschiedliche Realisierungen von Demokratie (Mittelwerte der Funktionen-, Prinzipien- und Demokratiequalitätswerte innerhalb jedes Clusters)

\begin{tabular}{lllll}
\hline & Cluster 1 & Cluster 2 & Cluster 3 & Cluster 4 \\
\hline Individuelle Freiheiten & 88,16 & $\mathbf{9 1 , 6 0}$ & 81,9 & 77,63 \\
Rechtsstaatlichkeit & $\mathbf{8 2 , 4 5}$ & 74,14 & 72,2 & 39,81 \\
Öffentlichkeit & 42,89 & $\mathbf{4 9 , 9 7}$ & 20,6 & 22,45 \\
Wettbewerb & 71,04 & $\mathbf{7 3 , 1 1}$ & 55,1 & 49,57 \\
Gewaltenkontrolle & 50,99 & 34,95 & 59,2 & $\mathbf{6 0 , 3 0}$ \\
Regierungs- und Implementierungsfähigkeit & 85,99 & $\mathbf{8 7 , 9 1}$ & 76,6 & 66,29 \\
Transparenz & $\mathbf{7 1 , 2 5}$ & 32,62 & 61,7 & 30,26 \\
Partizipation & $\mathbf{7 7 , 5 3}$ & 59,06 & 60,6 & 50,02 \\
Repräsentation & $\mathbf{7 7 , 6 2}$ & 53,93 & 56,9 & 58,61 \\
Freiheit & $\mathbf{7 3 , 8 6}$ & $\mathbf{7 6 , 8 8}$ & 49,8 & 39,35 \\
Kontrolle & $\mathbf{7 3 , 2 7}$ & 65,89 & 67,4 & 61,29 \\
Gleichheit & $\mathbf{8 2 , 6 9}$ & 46,61 & 63,3 & 41,19 \\
Demokratiequalität & $\mathbf{8 3 , 6 3}$ & 67,00 & 63,4 & 45,26 \\
\hline
\end{tabular}

fett höchster Mittelwert im Gruppenvergleich, schattiert drei Funktionen innerhalb der Gruppe mit stärkster Ausprägung, kursiv Funktion mit schwächster Betonung innerhalb der Gruppe

\subsection{Konstruktvalidität}

In unserem Demokratiekonzept unterscheiden wir neun Funktionen, welche die Prinzipien Freiheit, Gleichheit und Kontrolle umsetzen und deren Erfüllungsgrad schließlich die Demokratiequalität bestimmt. Wir vermuten, dass das Gewicht, welches eine Demokratie den unterschiedlichen Funktionen zumisst, zum einen ein Desiderat fortwährender politischer und gesellschaftlicher Deliberation, zum anderen aber auch ein Resultat historisch gewachsener Pfadabhängigkeiten ist. Wir erwarten deshalb unterschiedliche empirische Realisierungen von Demokratie, die in eine beachtliche variety of democracies mündet.

Diese Vermutung muss sich nun mit Hilfe des Demokratiebarometers empirisch nachweisen lassen. Mit Hilfe einer Clusteranalyse der 30 Länder für das Jahr 2005 lassen sich unterschiedliche Demokratiemuster finden (Tab. 2). ${ }^{19}$

Die Clusteranalyse unterteilt die Länder in vier Gruppen: in der ersten Gruppe finden sich Belgien, Dänemark, Finnland, Island, Neuseeland, die Niederlande, Norwegen und Schweden. Diese Gruppe weist die höchste mittlere Demokratiequalität sowie die höchsten mittleren Werte in den Prinzipien Kontrolle und Gleichheit auf. Auffällig ist insbesondere die starke Betonung des Prinzips, Gleichheit'. In der zweiten Gruppe werden die Kleinstaaten Luxemburg, die Schweiz und Zypern zusammengefasst. In dieser Gruppe wird das Prinzip Freiheit am stärksten und das Prinzip Gleichheit vergleichsweise schwach betont. Ersteres ist auf eine im Vergleich relativ starke Betonung der Funktion Öffentlichkeit und letzteres auf die schwache Betonung der Funktion Transparenz zurückzuführen. Australien, Deutschland, Frankreich, Großbritannien, Irland, Japan, Kanada,

19 Die Clusteranalyse wurde mit den Funktionenwerten aller Länder für das Jahr 2005 durchgeführt $(N=30)$. Als Methode wurde die gebräuchliche Ward-Methode mit quadrierter euklidischer Distanz angewendet. 
Österreich, Portugal, Slowenien, Spanien, Ungarn und die USA machen die zahlenmäßig größte Gruppe 3 aus. Die Länder dieser Gruppe scheinen Kontrolle stärker zu betonen als Gleichheit und Freiheit, wobei das Ungleichgewicht zwischen den drei Prinzipien nicht ganz so stark ist wie in Gruppe 2. Die vierte Gruppe schließlich umfasst die restlichen sechs Länder Costa Rica, Italien, Malta, Polen, die Tschechische Republik und Südafrika. In dieser Gruppe ist die mittlere Demokratiequalität im Jahr 2005 am geringsten. Auch hier wird Kontrolle am stärksten betont, diesmal allerdings recht deutlich. Sowohl Freiheit wie auch Gleichheit sind hier im Mittel am wenigsten stark ausgeprägt.

Die Ländergruppen können hinsichtlich der einzelnen Funktionen beschrieben werden. Auffallend ist, dass im Jahr 2005 die individuellen Freiheiten wie auch die Regierungs- und Implementierungsfähigkeit in allen vier Gruppen mit am besten erfüllt zu sein scheinen. Mit Ausnahme der Gruppe 4 zeigt sich die Rechtsstaatlichkeit als vergleichsweise gut entwickelt. In der Gruppe 4 scheint der Gewaltenkontrolle allerdings ein größeres Gewicht beigemessen zu werden. Öffentlichkeit hingegen ist in drei der vier Ländergruppen die am schlechtesten erfüllte Funktion - wenn auch auf unterschiedlichem Niveau. Ausnahme bildet hier die Gruppe 2, in der allerdings die Funktion der Transparenz vergleichsweise unerfüllt bleibt.

Selbstverständlich müssten die einzelnen Gruppen genauer analysiert, die Bedeutung der unterschiedlichen Erfüllungsgrade ausführlich erörtert und Analysen für die restlichen Jahre durchgeführt werden. Für den hier verfolgten Zweck reicht es aber, zu sehen, dass auf der Basis der Demokratiebarometerdaten die Idee der unterschiedlichen Realisierungen von Demokratie empirisch nachgezeichnet werden kann.

\subsection{Kriteriumsvalidität}

Eine weitere Möglichkeit, die Validität unseres Instruments zu testen, ist die Kriteriumsvalidität (Cassel und Lo 1997; Lawrence 2006; Wyckoff 1987). Hier wird untersucht, wie das Demokratiebarometer mit externen Variablen zusammenhängt, mit denen es aus theoretischen Gründen korrelieren müsste. So kann etwa vermutet werden, dass eine hohe Demokratiequalität mit einem hohen Entwicklungs- bzw. Wohlstandsniveau einhergeht. Unsere Analysen bestätigen dies: Die mittels des Demokratiebarometers bestimmte Demokratiequalität hängt positiv mit dem von der UNO bzw. vom UNDP entwickelten ,Human Development Index (HDI) zusammen. ${ }^{20}$ Pearsons $r$ beträgt über alle Jahre hinweg $0,54 .{ }^{21}$ Dies ist insbesondere auch deshalb bemerkenswert, weil der HDI in unserem Sample etablierter Demokratien nicht stark variiert (Abb.4). ${ }^{22}$

20 Der HDI setzt sich aus der Lebenserwartung, der Alphabetenrate, der Schülerquote sowie der realen Pro-Kopf-Kaufkraft zusammen.

21 Die Stärke der Korrelation variiert zwischen den Jahren: 1995: 0,57; 1998: 0,58; 2000: 0,59; 2001: 0,42; 2005: 0,58.

22 Der HDI reicht von 0 bis 1 . Die verfügbaren 150 ,blue print'-Länder-Jahre (30 Länder in den Jahren 1995, 1998, 2000, 2001 und 2005) weisen einen Mittelwert von 0,91 bei einer Standardabweichung von 0,05 auf. 


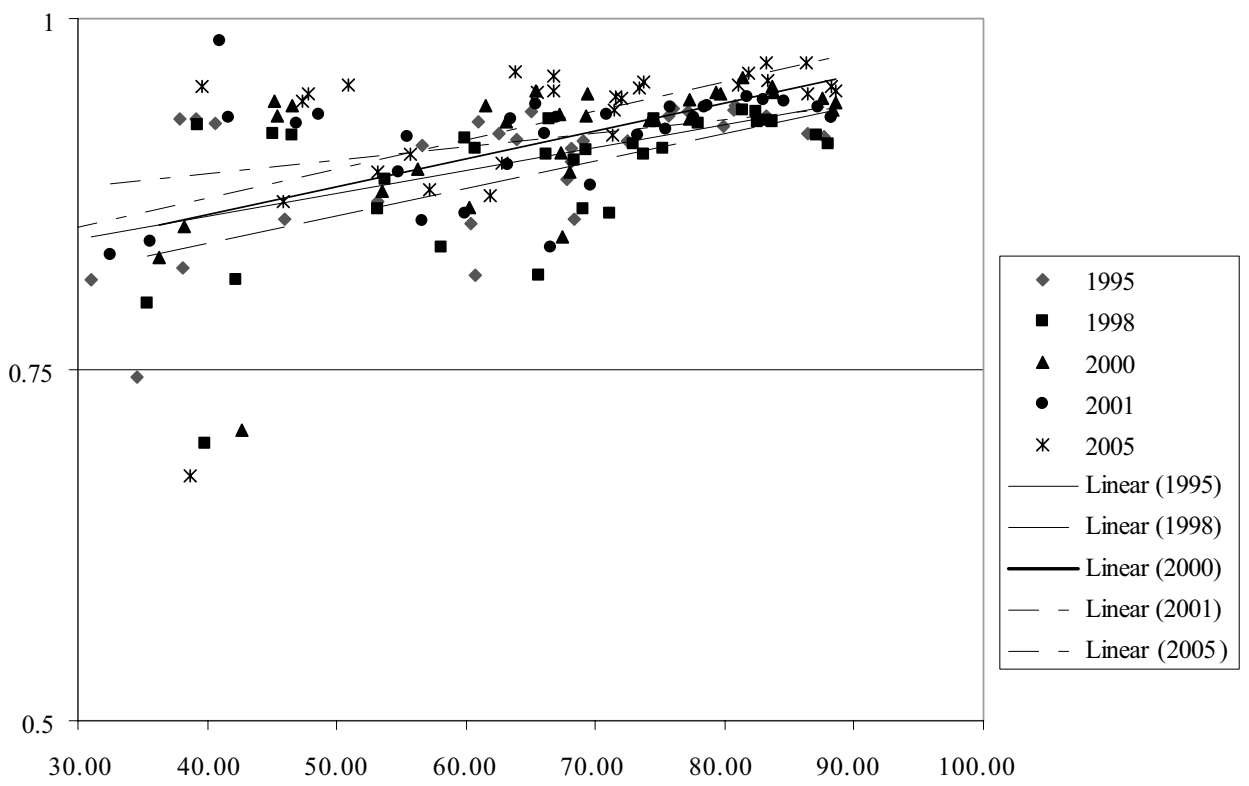

Abb. 4: Demokratiequalität und HDI ( $N=150$ (30 Länder in den Jahren 1995, 1998, 2000, 2001 und 2005); Quelle: Human Development Index)

\subsection{Endogene Validität}

Die Verknüpfung mit anderen Demokratiemessungen scheint sich in der empirischen Demokratieforschung als Standard-Proxytest zur Einschätzung der Messqualität eines Messinstruments etabliert zu haben (Bollen 1993; Bollen und Paxton 1998; Gaber 2000; Munck und Verkuilen 2002; Welzel 2000). Allerdings können für das Demokratiebarometer in der Form des ,blue print'-Samples keine Korrelationstests mit bestehenden Maßen durchgeführt werden, weil sich für diese Länder in bisherigen Indizes keine Varianz ergibt: sowohl Polity als auch Freedom House stufen die 30 Länder wie erwähnt durchwegs als etablierte Demokratien ein. Wir nutzen deshalb mit den „Worldwide Governance Indicators“ (Kaufmann et al. 2009) ein Maß, das zwar nicht Demokratiequalität, sondern ,good governance“ misst. Diese beiden Konzepte sind jedoch eng miteinander verwandt. ${ }^{23}$ In Abb. 5 sind die Koeffizienten der Korrelationen zwischen dem Governan-

23 Governance consists of ,the traditions and institutions by which authority in a country is exercised. This includes the process by which governments are selected, monitored and replaced, the capacity of the government to effectively formulate and implement sound policies and the respect of citizens and the state for the institutions that govern economic and social interactions among them" (Kaufmann et al. 2009, S. 5). 


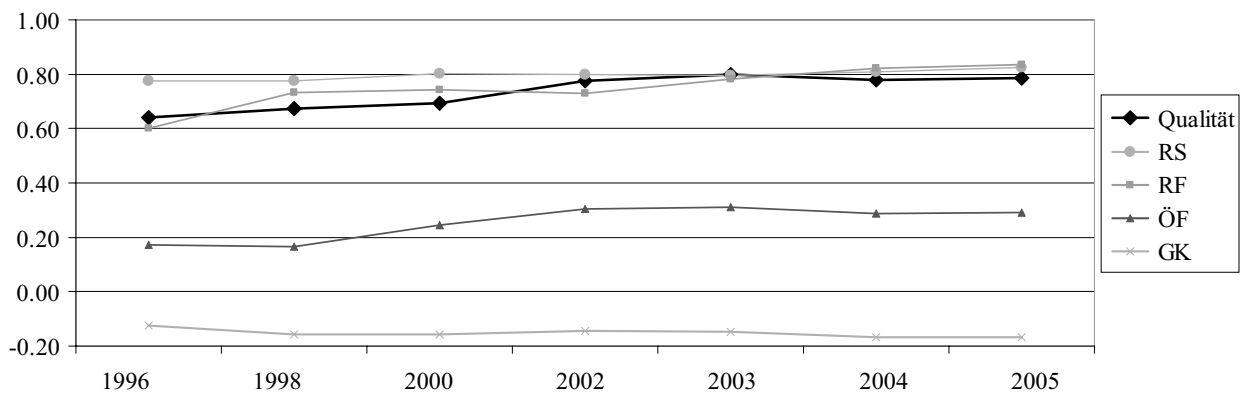

Abb. 5: Korrelationen zwischen Demokratiebarometer und Governance-Index. (Korrelationen zwischen aggregierten Governance-Indikatoren (s. Fussnote 24) und Demokratiequalität sowie den Funktionen ,Rechtsstaatlichkeit‘ $(R S)$, ,Regierungs- und Implementierungsfähigkeit‘ $(R F)$, Öffentlichkeit‘ $(O \ddot{O} F)$ und ,Gewaltenkontrolle‘ $(G K)$ für alle ,blue print'-Länder in den Jahren, in denen die Governance-Indikatoren erhältlich sind (1996, 1998, 2000, 2002, 2003, 2004, 2005)

ce-Index ${ }^{24}$ der Weltbank und der Demokratiequalität für jene Jahre abgetragen, für die Governance-Indikatoren erhältlich sind.

Es kann beobachtet werden, dass die Demokratiequalität insgesamt stark mit dem aggregierten Governance-Index korreliert. Dies kann als Indiz für die Messqualität des Demokratiebarometers gewertet werden. Zwei weitere Beobachtungen verdienen hier allerdings Erwähnung:

1. Der Zusammenhang zwischen dem aggregierten Governance-Index und einzelnen Funktionen variiert sehr stark. Schwache und sogar negative Korrelationen zeigen sich mit den Funktionen ,Gewaltenkontrolle“ (mittlere Pearsons Korrelationswerte von $-0,15)$, Öffentlichkeit‘ $(0,25)$, ,Repräsentation ‘ $(0,40)$, Individuelle Freiheiten ‘ $(0,44)$, und ,Wettbewerb` $(0,45)$; mittlere bis starke statistische Zusammenhänge finden sich zwischen dem aggregierten Governance-Index und den Funktionen ,Partizipation` $(0,55)$, ,Transparenz' $(0,63)$, ,Regierungs- und Implementierungsfähigkeit ‘ $(0,75)$, und ,Rechtsstaatlichkeit‘ $(0,80)$. Diesen Funktionen wird im GovernanceIndex der Weltbank besonderes Gewicht eingeräumt. Es scheint, als würden jene Funktionen eher schwach korrelieren, die sich primär dem Kontrollprinzip zuordnen lassen. Dies bestätigt die von uns geteilte Ansicht Lauths (2004), dass bisherige Demokratieindizes diesem Prinzip stiefmütterlich begegnen. Mit anderen Worten:

24 Die Worldwide Governance Indicators werden seit 1996 alle zwei Jahre und seit 2002 jährlich erhoben. Sie bestehen aus den sechs Dimensionen, Voice and Accountability‘ (Partizipationsmöglichkeiten), ,Political Stability“ (Wahrscheinlichkeit gewaltfreier Herrschaftswechsel), ,Government Effectiveness' (Qualität der Policies und Fehlen nicht-legitimierten Drucks), ,Regulatory Quality“ (Kapazität der Regierung, gute Policies zu produzieren), ,Rule of Law ‘ (Grad der Akzeptanz von Rechtsstaatlichkeit in der Gesellschaft) und ,Control of Corruption “ (Grad an Korruption), welche anhand von Indikatoren bestimmt werden, die aus verschiedenen und mit der Zeit zunehmenden Quellen gewonnen werden. Für die Korrelationen haben wir diese sechs Dimensionen für jedes Länderjahr per Mittelwert zu einem Gesamtscore aggregiert. 
ähnlich wie bestehende Demokratiemaße scheint auch der Governance-Index hinsichtlich demokratischer Kontrolle konzeptuell unterspezifiziert zu sein.

2. Die Stärke der Korrelationen zwischen der von uns gemessenen Demokratiequalität und dem aggregierten Governance-Index nimmt mit der Zeit zu. Dies ist darauf zurückzuführen, dass der Governance-Index 1996 weniger differenziert misst als 2005. ${ }^{25}$ Die Messung der Demokratiequalität bleibt hingegen für alle Jahre genau gleich. Dies spricht erneut für endogene Validität des Demokratiebarometers. Wir messen die feinen Unterschiede zwischen etablierten Demokratien und zwar während der gesamten Messperiode auf immer die gleiche Weise.

\section{Konklusion}

Zusammenfassend lässt sich festhalten, dass uns unsere informierte Intuition ${ }^{26}$ nicht getäuscht hat. Obwohl bisherige Demokratieindizes dafür ,blind' sind, gibt es deutliche Unterschiede in der Qualität etablierter Demokratien, die sich messen und darstellen lassen. Dies gilt nicht nur für die drei eingangs genannten Länder Italien, USA und Finnland, sondern für alle Staaten, welche in den elf Jahren von 1995 bis 2005 konstant als etablierte Demokratien gelten können. Mit Hilfe eines theoretisch breit abgestützten Konzepts, welches auf einer systematisch-deduktiven Logik basiert, sowie einem umfassenden Set an Indikatoren, die anhand klarer Regeln schrittweise aggregiert werden, gelingt es, diese Unterschiede deutlich sichtbar zu machen.

So hat sich gezeigt, dass etablierte Demokratien hinsichtlich ihrer gesamten Demokratiequalität stark variieren und dass sich die Demokratiequalität innerhalb eines Landes über die Zeit verändert. Insgesamt nimmt die Demokratiequalität zwischen 1995 und 2005 in rund zwei Dritteln der 30 hier untersuchten Staaten zu. Dies weist erstens darauf hin, dass von einer allseits beklagten Krise der Demokratie nicht die Rede sein kann. Zweitens macht der Befund deutlich, dass auch etablierte Demokratien noch erhebliches Entwicklungspotenzial haben. Ein Endstadium der Demokratisierung der Demokratie ist in diesem Sinne nicht in Sicht (Dahl 1971; Offe 2004).

Dies liegt nicht zuletzt daran, dass eine gleichzeitige Maximierung aller neun Demokratiefunktionen aufgrund eines gewissen Spannungsverhältnisses zwischen den Prinzipien ,Freiheit‘ und ,Gleichheit‘ kaum möglich ist. Entsprechend bestätigen die Netzdiagramme (vgl. Abb.2), dass Demokratie das Produkt fortwährender gesellschaftlicher und politischer Aushandlungsprozesse ist und es deshalb verschiedene empirische Realisierungen von Demokratien gibt, die die drei Prinzipien und neun Funktionen unterschiedlich gewichten bzw. erfüllen. So lassen sich verschiedene Demokratiemuster

25 Tatsächlich nimmt die Zahl der Indikatoren, die in die jeweiligen Dimensionen des GovernanceIndex einfließt bis 2005 kontinuierlich zu. Mit anderen Worten: die Basis des Weltbank-Maßes wird mit der Zeit breiter und damit auch unserem Demokratieindex ähnlicher.

26 Intuition wird hier nicht als vorrationale Annahme verwendet, sondern als eine Annahme, die sich aus vielen partikulären Demokratiebeobachtungen in der wissenschaftlichen Debatte speist. 
identifizieren, die zudem Aufschluss über spezifische Defizite bzw. Reform- oder Verbesserungspotenziale in einzelnen Ländern geben können.

Hierfür sind aber noch differenziertere Analysen erforderlich. ${ }^{27}$ Das Demokratiebarometer bietet vielfältige Möglichkeiten für diverse Kausalanalysen. Entsprechend ist geplant, die Ursachen wie Auswirkungen unterschiedlicher Demokratiequalitäten auszuloten. Einerseits soll untersucht werden, wie die Demokratiequalität von unterschiedlichen Institutionenordnungen beeinflusst wird. Andererseits kann getestet werden, welche wirtschaftlichen oder gesellschaftlichen Auswirkungen eine hohe Demokratiequalität hat und ob es einen Zusammenhang zwischen Qualität und Leistungsstärke einer Demokratie in der Politikproduktion gibt. Das Demokratiebarometer stellt für all diese Kausalanalysen die nötigen Daten bereit.

Freilich kann die Komplexität des Demokratiebarometers mit seinen 100 Indikatoren und spezifizierten Messverfahren kritisiert werden. Darüber hinaus ist das Demokratiebarometer - wie jedes andere sozialwissenschaftliche Instrument - von der Qualität seiner Indikatoren abhängig. Auch hier gilt also das Bonmot Einsteins: „Not everything that can be counted counts, and not everything that counts can be counted". So können bisher einige der Komponenten nicht ganz befriedigend mit Proxies gemessen werden, da (noch) keine besseren Daten bestehen. Allerdings zeigen erste Tests, dass das Instrument durchaus als valide und reliabel betrachtet werden kann. Zudem darf Sparsamkeit nicht auf Kosten einer adäquaten Messung des komplexen Phänomens ,Demokratie‘ gehen. Ebenfalls ist hervorzuheben, dass mit dem vorliegenden neuen Instrument versucht wurde, Fehler und Schwächen bisheriger Demokratiemessungen zu vermeiden. Nicht nur das theoretische Konzept, das über bisherige, v. a. minimalistische und theoretisch kaum begründete Entwürfe weit hinaus geht, sondern die methodischen Überlegungen, die theoretisch fundiert und empirisch abgesichert werden, geben dem Demokratiebarometer eine solide Basis, die weit über das hinausgeht, was an Demokratiemessungen bisher vorgelegt wurde.

\section{Anhang}

\section{Verwendete Indikatoren}

Im Folgenden werden pro Funktion und Komponente die im Demokratiebarometer enthaltenen Indikatoren aufgelistet. Die Beschreibung der Indikatoren ist jedoch nur grob und in englischer Sprache. Ein detailliertes Codebuch ist unter www.democracybarometer.org erhältlich.

27 Ziel unserer weiterführenden Forschung ist es unter anderem, mit Hilfe von umfassenden Untersuchungen die Qualität etablierter Demokratien im Quer- und Längsschnitt für ein Sample von rund 75 Ländern detailliert auszuleuchten und unterschiedliche Demokratiemuster zu bestimmen. 


\section{Individuelle Freiheiten}

1. Komponente ,Recht auf körperliche Unversehrtheit':

1a) Subkomponente, Verfassungsrechtlich garantiertes Recht auf körperliche Unversehrtheit":

- Consttort: Constitutional ban of torture or cruel or unusual punishment.

- Convtort: Ratification of Convention Against Torture and Other Cruel, Inhuman or Degrading Treatment or Punishment.

1b) Subkomponente ,Keine Eingriffe durch den Staat":

- Politterr: Political Terror Scale; degree of political terror by government.

- Torture: Torture and other cruel, inhumane, or degrading treatment or punishment.

1c) Subkomponente, Gegenseitige Akzeptanz des Rechts auf Unversehrtheit in der Bevölkerung':

- Homicide: Number of homicides per 100.000 capita, standardized according to best value in overall sample (reversed).

- Riot: Number of violent demonstration or clash of more than 100 citizens involving the use of physical force (reversed).

2. Komponente ,Recht auf Selbstbestimmung':

2a) Subkomponente ,Verfassungsrechtlich garantierte Freiheit der individuellen Lebensgestaltung':

- Constrel: Existence of constitutional provisions protecting religious freedom.

- Constfreemov: Existence of constitutional provisions guaranteeing freedom of movement.

2b) Subkomponente, Recht auf freie Lebensgestaltung :

- Freerelig: Extent to which the freedom of citizens to exercise and practice their religious beliefs is subject to actual government restrictions.

- Freemove: Extent of citizens' freedom to travel within their own country and to leave and return to that country.

2c) Subkomponente ,Effektive Eigentumsrechte“:

- Propright: Degree to which a country's laws protect private property rights and the degree to which its government enforces those laws.

- Secprop: Personal security and private property are adequately protected. 


\section{Rechtsstaatlichkeit}

1. Komponente, Gleichheit vor dem Gesetz :

1a) Subkomponente ,Verfassungsmässig garantierte Unparteilichkeit von Gerichten":

- Constfair: Constitutional provisions for fair organisation of court system (no exceptional courts and hierarchical judicial system).

- Pubtrial: Existence of constitutional provisions guaranteeing a public trial.

1b) Subkomponente ,Effektive Unabhängigkeit der Judikative“:

- Judindepcor: Assessment of the level of independence of the judiciary (no corruption within or pressures from outside the judiciary)

- Judindepinf: Business people's assessment of the level of independence of the judiciary from political influences of the government, citizens or firms.

1c) Subkomponente, Effektive richterliche Objektivität':

- Impcouts: Impartial Courts: Business people's assessment of statement: „,The legal framework in your country for private businesses to settle disputes and challenge the legality of government actions and/or regulations is inefficient and subject to manipulation“".

- Intgrlegal: Integrity of the legal system.

2. Komponente, Qualität der Justiz':

2a) Subkomponente ,Verfassungsmässig garantierte Professionalität der Gerichte“:

- Profjudge: Professionalism (law degree, professional experience) is a precondition for appointment of judges to highest courts.

- Proftenure: Length of judges' tenure.

2b) Subkomponente, Vertrauen in die Justiz':

- Confjust: Confidence in the legal system: share of survey respondents indicating high confidence/trust.

- Fairjust: Business people's assessment of the confidence in the fair administration of justice in the society.

2c) Subkomponente, Vertrauen in die Polizei":

- Confpolice: Confidence in the police: share of survey respondents indicating high confidence/trust.

- Fairpolice: Business people's assessment of the reliability/effectiveness of the police services. 


\section{Öffentlichkeit}

1. Komponente, Vereinigungsfreiheit":

la) Subkomponente ,Verfassungsmässig garantierte Vereinigungsfreiheit':

- Constfras: Existence of constitutional provisions guaranteeing freedom of association.

- Constass: Existence of constitutional provisions guaranteeing freedom of assembly.

1b) Subkomponente, Vereinigungsgrad ökonomischer Interessen ':

- Union: Trade union density.

- Memproorg: Share of survey respondents indicating that they are member in a professional organization.

1c) Subkomponente, Vereinigungsgrad öffentlicher Interessen':

- Memhuman: Share of survey respondents indicating that they are member in and/or actively spend time for a humanitarian organization.

- Memenviron: Share of respondents indicating that they are member in and/or actively spend time for an environmental or animal rights organization.

2. Komponente, Meinungsfreiheit ${ }^{6}$ :

2a) Subkomponente ,Verfassungsmässig garantierte Meinungsfreiheit‘:

- Constspeech: Existence of constitutional provisions guaranteeing freedom of speech.

- Constpress: Existence of constitutional provisions guaranteeing freedom of press.

2b) Subkomponente, Medienangebot":

- Newsimp: Import of newspapers, journals and periodicals as a \% of GDP.

- Newspaper: Number of daily newspapers (titles) per 1 mio. inhabitants.

2c) Subkomponente, Politische Neutralität des Pressesystems ':

- Balpress: Ideological balance of the press system (regional and national newspapers).

- Neutrnp: Share of neutral/independent newspapers' circulation (weighted by frequency of publication) of a country's total newspaper circulation.

\section{Wettbewerb}

1. Komponente, Vulnerability“:

1a) Subkomponente ,Formelle Regeln für Konkurrenz':

- Meandistrict: Mean district magnitude.

- Gerryman: Gerrymandering: extent to which changing electoral districts is possible (reversed). 
1b) Subkomponente, Knappheit des Wahlresultates :

- Largepavo: Margin of electoral concentration of votes $=100 \%$-pstrongest, where pstrongest $=$ percentage of votes obtained by strongest party.

- Votediff: 100-Difference between largest and second largest lower house party in \% of all votes.

1c) Subkomponente, Geringe Sitzkonzentration ':

- Herfindex: Herfindahl index: the sum of the squared seat shares of all parties in parliament. Measures the degree of concentration of seats on single or few parties (reversed).

- Seatdiff: 100-Difference between largest and second largest lower house party in \% of all seats.

2. Komponente, Contestability“:

2a) Subkomponente ,Niedrige Wahlhürden':

- Adminhurd: Degree/number of administrative requirements to become a competitor (reversed).

- Legthresh: No or low legal electoral threshold, calculated as 100-legal threshold.

2b) Subkomponente ,Effektive Wettbewerbschancen“:

- Smallpavo: Chance for small parties to win a seat: share of votes of smallest party in national parliament (reversed).

- Nuparties: Number of important parties ( $>1 \%$ of votes) running for elections.

\section{2c) Subkomponente ,Effektive Eintrittschancen':}

- Enep: Effective number of parties at the electoral level.

- Effparrat: Ratio of effective number of parties at the parliamentary level and the effective number of parties at the electoral level.

\section{Gewaltenkontrolle}

1. Komponente ,Beziehung zwischen Exekutive und Legislative“:

1a) Subkomponente, Institutionalisierte Absetzungsmöglichkeiten für Exekutive und Legislative":

- Controlle: Constitutionally guaranteed possibility for executive to veto laws passed by the legislature and to dissolve the legislature.

- Controlex: Constitutionally guaranteed possibility for legislative branch to remove executive from office (instruments such as vote of no confidence/impeachment as well as difficulty to proceed). 
1b) Subkomponente,Ausgeglichene Machtbalance zwischen Exekutive und Legislative':

- Balpowexle: Balance of powers (opposition vs. government) according to Altman und Perez-Liñan 2002.

- Seatsgov: 100-Proportion of parliamentary seats belonging to governing parties.

2. Komponente, Weitere institutionelle Sicherungen':

2a) Subkomponente, Verfassungsgerichtsbarkeit':

- Judrev: Extent to which Supreme or Constitutional Court judges have the power to review the constitutionality of (proposed) laws.

- Powjudi: Extent of constitutional provisions guaranteeing judicial exclusive authority over political decisions.

2b) Subkomponente ,Föderalismusgrad':

- Federgeta: Degree of federalism according to Geering-Thacker (2004).

- Nonunitar: Extent of unitarism (combination of nonfederalism and nonbicameralism)

2c) Subkomponente ,Subnationale fiskale Autonomie‘:

- Subexp: Subnational expenditures as a \% of total state expenditures.

- Subrev: Subnational revenues as a \% of GDP.

Regierungs- und Implementierungsfähigkeit

1. Komponente, Ressourcen':

1a) Subkomponente ,Zeithorizont":

- Legislen: Length of legislative period

- Govterm: Length of government term.

1b) Subkomponente, Öffentliche Unterstützung :

- Confgov: Confidence in the government: share of survey respondents indicating high confidence/trust.

- Devbehav. Share of citizens which do not endorse behavior and attitudes that are directed against the democratic society (justifying claiming government benefits/ avoiding a fare on public transport/cheating on taxes/someone accepting a bribe).

1c) Subkomponente ,Regierungsstabilität':

- Govstab: Stability of the government: no changes during legislature.

- Cabchange: Number of major cabinet changes (reversed). 
2. Komponente, Bedingungen zur effizienten Implementierung ${ }^{6}$ :

2a) Subkomponente ,Keine regierungsfeindlichen Aktionen':

- Antigovact: No legitimate and peaceful political anti-government action (reversed sum of number of strikes aimed at national government policies or authority and number of peaceful gatherings for the primary purpose of displaying or voicing their opposition to government policies or authority).

- Violantig: Not illegitimate and violent political anti-government action (reversed sum of number of armed activities, sabotage, or bombings carried on by independent bands of citizens or irregular forces and aimed at the overthrow of the present regime and number of illegal or forced changes in the top government elite, any attempt at such a change, or any successful or unsuccessful armed rebellion whose aim is independence from the central government).

2b) Subkomponente, Keine Einmischung :

- MipRip: No political interference by military and religious authorities.

- Publser: Independence of public service of political interference.

2c) Subkomponente ,Durchsetzungsfähigkeit der Verwaltung ‘:

- Govdec: Business people's assessment of effectiveness of implementation of government decisions.

- Bureau: Assessment of the strenght and expertise of the bureaucracy to govern withouth drastic changes in policy or interruptions in government services as well as its autonomy from political pressure.

\section{Transparenz}

1. Komponente ,Absenz von Geheimhaltung':

1a) Subkomponente ,Keine Korruption':

- Bribcorr: Business people's assessment of the prevalence of bribery and corruption.

- CPI: Corruption Perception Index (Transparency International): overall extent of corruption (frequency and/or size of bribes) in the public and political sectors.

1b) Subkomponente, Offenlegung von Parteifinanzierung ':

- Discinco: Existence of provision for disclosure of income by political parties.

- Discexp: Existence of provision for public disclosure of expenditure by political parties

2. Komponente ,Voraussetzungen für transparenten politischen Prozess ‘:

2a) Subkomponente ,Bereitschaft zur transparenten Kommunikation ':

- Transp: Business people's assessment of the transparency of government policy. 
2b) Subkomponente, Informationsfreiheitsgesetzgebung :

- RestricFOI: Restriction of freedom of information/barriers for access to official information.

- EffFOI: Effectiveness of Freedom of Information laws.

2c) Subkomponente, Offene und freie politische Berichterstattung :

- Legmedia: Press Freedom: Legal Environment: The examination of both the laws and regulations that could influence media content and the government's inclination to use these laws and legal institutions to restrict the media's ability to operate.

- Polmedia: Press Freedom: Political Environment: the degree of political control over the content of news media.

\section{Partizipation}

1. Komponente, Gleichheit der Partizipation':

1a) Subkomponente ,Nicht-Selektivität der Wahlpartizipation':

- Repturnined: Representative voter turnout (parliamentary elections) in terms of resources (no participation gap in terms of education and income).

- Repturngeag: Representative voter turnout (parliamentary elections) in terms of gender and age (no participation gap).

1b) Subkomponente, Nicht-Selektivität alternativer Partizipation :

- Repaltined: Representative alternative participation (signing petitions, attending lawful demonstrations) in terms of resources (no participation gap).

- Repaltgeag: Representative alternative participation (signing petitions, attending lawful demonstrations) in terms of gender and age (no participation gap).

1c) Subkomponente ,Partizipationsrechte':

- Suffrage: Extent of universal active suffrage.

- Regprovap: Registered voters as a \% of the voting age population.

2. Komponente, Effektive Partizipation':

2a) Subkomponente, Effektive institutionalisierte Partizipation ':

- Turnout: Mean level of participation in \% of registered electorate in legislative and/or presidential elections and/or national referenda.

2b) Subkomponente ,Effektive nicht-institutionalisierte Partizipation':

- Petitions: Share of survey respondents indicating having signed petitions.

- Demons: Share of WVS respondents indicating having attended lawful demonstrations. 
2c) Subkomponente, Gesetzliche Grundlagen zur erleichterten Stimmabgabe :

- Facilitat: Extent of facilitated voting.

- Regist: Voter registration is not compulsory.

\section{Repräsentation}

1. Komponente ,Substantielle Repräsentation“:

1a) Subkomponente ,Disproportionalität':

- Gallagindex: Index of proportionality according to Gallagher (vote-seat congruence).

- Issuecongr: Congruence between left-right position of voters and left-right position of parliamentarians (measured by party's positions).

1b) Subkomponente ,Strukturelle Opportunitäten substantieller Repräsentation:

- Seatperin: Number of parlimentary seats (lower house) per inhabitants.

- Dirdem: opportunities for vertical control on political decisions. Availability of mandatory and facultative referenda (including their coverage, terms of adoption, bondage, initiator, popular vote, hurdles).

2. Komponente ,Deskriptive Repräsentation“:

2a) Subkomponente ,Effektiver Zugang zu politischen Ämtern für Minderheiten :

- Accpowmin: Access to power for minority groups (average of all groups in a country).

- Poldismin: Index of political discrimination of minority groups concerning equal representation (reversed).

2b) Subkomponente ,Adäquate Frauenvertretung“:

- Womrep: Number of female representatives in the lower house of parliament in $\%$ of all seats.

- Womgov: Share of female members of the government.

2c) Subkomponente, Spezifische politische Frauenrechte':

- Polrightwom: Degree of political rights for women, including the right to vote, the right to run for political office, the right to hold elected and appointed government positions, the right to join political parties, and the right to petition government officials.

- Constraints: Extent of universal passive suffrage. 


\section{Literatur}

Abromeit, Heidrun. 2004. Die Messbarkeit von Demokratie: Zur Relevanz des Kontextes. Politische Vierteljahresschrift 45 (1): 73-93.

Alonso, Sonia. 2010. Multinational democracy and the comsequences of compounded representation: The case of Spain. In The future of representative democracy, Hrsg. Sonia Alonso, John Keane und Wolfgang Merkel. Cambridge: Cambridge University Press (i.E.).

Alonso, Sonia, John Keane, und Wolfgang Merkel, Hrsg. 2010. The future of representative democracy. Cambridge: Cambridge University Press.

Altman, David, und Aníbal Pérez-Liñán. 2002. Assessing the quality of democracy: Freedom, competitiveness and participation in eighteen Latin American countries. Democratization 9 (2): $85-100$.

Alvarez, Michael, José Antonio Cheibub, Fernando Limongi, und Adam Przeworski. 1996. Classifying political regimes. Studies on Comparative International Development 31 (2): 1-37.

Arat, Zehra F. 1991. Democracy and human rights in developing countries. Boulder: Rienner.

Banducci Susan A., Todd Donovan, und Jeffrey A. Karp. 2004. Minority representation, empowerment, and participation. The Journal of Politics 66 (2): 534-556.

Barber, Benjamin R. 1984. Strong democracy: participatory politics for a new age. Berkeley: University Press.

Bartolini, Stefano. 1999. Collusion, competition, and democracy. Journal of Theoretical Politics 11 (4): 435-470.

Bartolini, Stefano. 2000. Collusion, competition and democracy: Part II. Journal of Theoretical Politics 12 (1): 33-65.

Beetham, David. 2004. Freedom as the foundation. Journal of Democracy 15 (4): 61-75.

Beierwaltes, Andreas. 2000. Demokratie und Medien: Der Begriff der Öffentlichkeit und seine Bedeutung für die Demokratie in Europa. Baden-Baden: Nomos.

Berg-Schlosser, Dirk. 1999. Empirische Voraussetzungen und allgemeine Konstituierungsbedingungen von Demokratie. In Perspektiven der Demokratie. Probleme und Chancen im Zeitalter der Globalisierung, Hrsg. Dirk Berg-Schlosser und Hans-Joachim Giegel, 57-81. Frankfurt a. M.: Campus.

Berg-Schlosser, Dirk. 2004a. Indicators of democracy and good governance as measures of the quality of democracy in Africa: A critical appraisal. Acta Politica 39:248-278.

Berg-Schlosser, Dirk. 2004b. The quality of democracies in Europe as measured by current indicators of democratization and good governance. Journal of Communist Studies and Transition Politics 20 (1): 28-55.

Berlin, Isaiah. 2006. Freiheit. Vier Versuche. Frankfurt a. M.: Fischer.

Blais, André, Louis Massicotte, und Antoine Yoshinaka. 2001. Deciding who has the right to vote: A comparative analysis of election laws. Electoral Studies 20 (1): 41-62.

Böckenförde, Ernst Wolfgang. 1991. Staat, Verfassung, Demokratie. Studien zur Verfassungstheorie und zum Verfassungsrecht. Frankfurt a. M.: Suhrkamp.

Bollen, Kenneth A. 1990. Political democracy. Conceptual and measurement traps. Studies in Comparative International Development 25 (2): 7-24.

Bollen, Kenneth A. 1993. Liberal democracy. Validity and method factors in cross-national measures. American Journal of Political Science 37 (4): 1207-1230.

Bollen, Kenneth A., und Pamela Paxton. 1998. Detection and determinants of bias in subjective measures. American Sociological Review 63 (3): 465-478.

Bollen, Kenneth A., und Pamela Paxton. 2000. Subjective measures of liberal democracy. Comparative Political Studies 33:58-86.

Bovens, Mark. 2007. Analysing and assessing accountability: A conceptual framework. European Law Journal 13 (4): 447-468. 
Bühlmann, Marc, und Ruth Kunz. 2011. Confidence in the judiciary. A cross country comparison of independence and legitimacy of judicial systems. West European Politics 34 (2): 317-345.

Bühlmann, Marc, Wolfgang Merkel, Lisa Müller, und Bernhard Weßels. 2008. Wie lässt sich Demokratie am besten messen? Zum Forumsbeitrag von Thomas Müller und Susanne Pickel. Politische Vierteljahresschrift 49 (1): 114-122.

Camp Keith, Linda. 2002. Constitutional provisions for individual human rights (1977-1996): Are they more than mere ,Window Dressing?‘. Political Research Quarterly 55 (1): 111-143.

Cassel, Carol A., und Celia C. Lo. 1997. Theories of political literacy. Political Behavior 19 (4): 317-335.

Chanley, Virginia A., Thomas J. Rudolph, und Wendy M. Rahn. 2000. The origins and consequences of public trust in government: A time series analysis. The Public Opinion Quarterly 64 (3): 239-256.

Cingranelli, David L., und David L. Richards. 1999. Respect for human rights after the end of the cold war. Journal of Peace Research 36 (5): 511-534.

Cohen, Joshua, und Archon Fung. 2004. Radical democracy. Swiss Political Science Review 10 (4): 169-180.

Collier, David, und Steven Levitsky. 1997. Democracy with adjectives: Conceptual innovation in comparative research. World Politics 49 (3): 430-451.

Coppedge, Michael, und Wolfgang Reinicke. 1990. Measuring polyarchy. Studies in Comparative International Development XXV:51-72.

Crozier, Michel, Samuel P. Huntington, und Joji Watanuki, Hrsg. 1975. The crisis of democracy: Report on the governability of democracies to the trilateral commission. New York: New York University Press.

Dahl, Robert A. 1956. A preface to democratic theory. Chicago: University of Chicago Press.

Dahl, Robert A. 1971. Polyarchy. Participation and opposition. New Haven: Yale University Press.

Dahl, Robert A. 1976. Vorstufen zur Demokratie-Theorie. Tübingen: Mohr.

Dahl, Robert A. 1998. On democracy. New Haven: Yale University Press.

Dahl, Robert A. 2006. On political equality. New Haven: Yale University Press.

Dahlerup, Trude. 2010. Engendering representation. In The future of representative democracy, Hrsg. Sonia Alonso, John Keane und Wolfgang Merkel. Cambridge: Cambridge University Press (i.E.).

de la Porta, Rafael, Florencio Lopez-de-Silanes, Cristian Pop-Eleches, und Andrei Shleifer. 2004. Judicial checks and balances. Journal of Political Economy 112 (2): 445-470.

Diamond, Larry, und Leonardo Morlino. 2004. The quality of democracy: An overview. Journal of Democracy 15 (4): 14-25.

Elkins, D. J. 1974. The measurement of party competition. American Political Science Review 68:682-700.

Esquith, Stephen L. 1999. Toward a democratic rule of law: East and West. Political Theory 27 (3): 334-356.

Etzioni, Amitai. 1968. The active society. London: Collier-McMillan.

Fenske, Hans, Dieter Mertens, Wolfgang Reinhard, und Klaus Rosen. 1994. Geschichte der politischen Ideen. Von Homer bis zur Gegenwart. Frankfurt a. M.: Fischer.

Fishkin, James. 1991. Democracy and deliberation: New directions for democracy reform. New Haven: Yale University Press.

Fraenkel, Ernst, Hrsg. 1962. Staat und Politik. Frankfurt a. M.: Fischer.

Fraenkel, Ernst. 1991. Deutschland und die westlichen Demokratien. Frankfurt a. M.: Suhrkamp.

Gaber, Rusanna. 2000. Demokratie in quantitativen Indizes. Ein mehr- oder eindimensionales Phänomen? In Demokratiemessung. Konzepte und Befunde im internationalen Vergleich, Hrsg. Hans-Joachim Lauth, Gert Pickel und Christian Welzel, 112-131. Opladen: Westdeutscher.

Gasiorowski, Mark J. 1990. The political regimes project. Studies in International Development 25 (1): 109-125. 
Gastil, Raymond Duncan. 1990. The comparative survey of freedom: Experiences and suggestions. Studies in Comparative International Development 1:25-50.

Geering, John und Strom C. Thacker. 2004. Political institutions and corruption. The role of unitarism and parliamentarism. British Journal of Political Science 34: 295-330.

Gibson, James L. 2006. Judicial institutions. In Political institutions, Hrsg. R.A.W. Rhodes, Sarah A. Binder und Bert A. Rockman, 514-534. Oxford: Oxford University Press.

Gosepath, Stefan. 2007. Equality. Stanford encyclopedia of philosophy. http://plato.Stanford.edu/ entries/equality/. Zugriff am 17.7.2012.

Graber, Doris. 2003. The media and democracy: Beyond myths and stereotypes. Annual Review of Political Science 6:139-160.

Graber, Doris. 2004. Mediated politics and citizenship in the twenty-first century. Annual Review of Psychology 55:545-571.

Guillén, Mauro F. 2003. Is globalization civilizing, destructive or feeble? A critique of five key debates in the social science literature. Annual Review of Sociology 27:235-60.

Habermas, Jürgen. 1990 [1962]. Strukturwandel der Öffentlichkeit. Untersuchungen zu einer Kategorie der bürgerlichen Gesellschaft. Frankfurt: Suhrkamp.

Habermas, Jürgen. 1992. Faktizität und Geltung: Beiträge zur Diskurstheorie des Rechts und des demokratischen Rechtsstaates. Frankfurt a. M.: Suhrkamp.

Hadenius, Axel. 1992. Democracy and development. Cambridge: Cambridge University Press.

Hamilton, Alexander, und James Madison. 1993 [1788]. Die Federalist Papers. Darmstadt.

Harmel, Robert, und John D. Robertson. 1986. Government stability and regime support: A crossnational analysis. The Journal of Politics 48 (4): 1029-1040.

Held, David. 2006. Models of democracy. 3. Aufl. Cambridge: Polity Press.

Heller, Hermann. 1934. Staatslehre (Herausgegeben von Gerhart Niemeyer). Leiden: A. W. Sijthoff's Uitgeversmaatschappij N. V.

Heller, Herrmann. 1971. Gesammelte Schriften (2 Bd.). Leiden: Sijthoff.

Höffe, Ottfried. 1999. Demokratie im Zeitalter der Globalisierung. München: Beck.

Holden, Matthew. 2006. Exclusion, inclusion, and political institutions. In Political institutions, Hrsg. R.A.W. Rhodes, Sarah A. Binder und Bert A. Rockman, 163-190. Oxford: Oxford University Press.

Humana, Charles. 1992. World human rights guide. 3. Aufl. New York: Oxford University Press.

Islam, Roumeen. 2006. Does more transparency go along with better governance? Economics and Politics 18 (2): 121-167.

Jaggers, Keith, und Ted R. Gurr. 1995. Transition to democracy. Tracking the third wave with polity III indicators of democracy and autocracy. Journal of Peace Research 32 (4): 469-482.

Kant, Immanuel. 1902 [1785]. Grundlegung zur Metaphysik der Sitten. In Kants Gesammelte Schriften, Hrsg. Preußische Akademie der Wissenschaften. Berlin: Walter de Gruyter.

Kaufmann, Daniel, und Aart Kraay. 2008. Governance indicators: Where are we, where should we be going? MPRA Paper 8212.

Kaufmann, Daniel, Aart Kraay, und Massimo Mastruzzi. 2009. Governance matters VIII: Aggregate and individual governance indicators, 1996-2008. World Bank Policy Research Working Paper No. 4978.

Kelsen, Hans. 1925. Allgemeine Staatslehre. Enzyklopädie der der Rechts- und Staatswissenschaften. 23. Bd. Berlin: Springer.

Lauth, Hans-Joachim. 2004. Demokratie und Demokratiemessung. Eine konzeptionelle Grundlegung für den interkulturellen Vergleich. Wiesbaden: VS Verlag für Sozialwissenschaften.

Lawrence, Christopher N. 2006. Should voters be encyclopedias? Measuring the political sophistication of survey respondents. Saint Louis. http://polmeth.wustl.edu/retrieve.php?id=664. Zugegriffen: 8. Mai 2009.

Lijphart, Arend. 1997. Unequal participation: democracy's unresolved dilemma. American Political Science Review 91 (1): 1-14. 
Lindstedt Catharina. 2005. Transparency and corruption. The conditional significance of a free press. Paper prepared for the conference , The Quality of Government: What It Is, How to Get It, Why It Matters “, November 17-19 2005. The quality of government institute, department of political science, Göteborg University.

Linz, Juan J., und Alfred Stepan. 1996. Problems of democratic transition and consolidation: Southern Europe, South America and post-communist Europe. Baltimore: The Johns Hopkins University Press.

Locke, John. 1974 [1689]. Über die Regierung. Stuttgart: Reclam.

Manin, Bernhard, Adam Przeworski, und Susan C. Stokes. 1999. Elections and representation. In Democracy, accountability, and representation, Hrsg. Adam Przeworski, Susan C. Stokes und Bernhard Manin, 29-54. Cambridge: Cambridge University Press.

Mansbridge, Jane. 1999. Should blacks represent blacks and women represent women? A contingent yes. Journal of Politics 61 (3): 628-657.

Mansbridge, Jane. 2003. Rethinking representation. American Political Science Review 97 (4): $515-528$.

Merkel, Wolfgang. 2010. Systemtransformation. Eine Einführung in die Theorie und Empirie der Transformationsforschung. Wiesbaden: VS Verlag für Sozialforschung.

Merkel, Wolfgang, Hans-Jürgen Puhle, Aurel Croissant, Claudia Eicher, und Peter Thiery. 2003. Defekte Demokratien. Band 1: Theorie. Opladen: Leske und Budrich.

Meyer, Thomas. 2005. Theorie der Sozialen Demokratie. Wiesbaden: VS Verlag für Sozialwissenschaften.

Meyer, Thomas. 2009. Was ist Demokratie? Eine diskursive Einführung. Wiesbaden: VS Verlag für Sozialwissenschaften.

Mill, John Stuart. 1991 [1861]. Considerations on representative government. Amherst: Prometheus Books.

Miller, S. M., Martin Rein, Pamela Roby, und Bertram M. Gross. 1967. Poverty, inequality, and conflict. Annals of the American Academy of Political and Social science 373 (2): 16-52.

Montesquieu, Charles-Louis de Secondat, Baron de la Brède et de Montesquieu. 1965 [1748]. Vom Geist der Gesetze. Stuttgart: Reclam.

Morlino, Leonardo. 2004a. ,Good' and ,Bad' democracies: How to conduct research into the quality of democracy. Journal of Communist Studies and Transition Politics 20 (1): 5-27.

Morlino, Leonardo. 2004b. What is a ,Good' democracy? Democratization 11 (5): 10-32.

Müller, Thomas, und Susanne Pickel. 2007. Wie lässt sich Demokratie am besten messen? Zur Konzeptqualität von Demokratie-Indizes. Politische Vierteljahresschrift 48 (3): 511-539.

Munck, Gerardo L., und Jay Verkuilen. 2002. Conceptualizing and measuring democracy: Evaluating alternative indices. Comparative Political Studies 35 (1): 5-34.

Nida-Rümelin, Julian. 2006. Demokratie und Wahrheit, München: Beck.

Norris, Pippa. 2000. A Virtuous circle: Political communications in post-industrial societies. Cambridge: Cambridge University Press.

O’Donnell, Guillermo. 1994. Delegative democracy. Journal of Democracy 5 (1): 55-70.

O'Donnell, Guillermo. 2004. Why the rule of law matters. Journal of Democracy 15 (4): 32-46.

Offe, Claus, Hrsg. 2004. Demokratisierung der Demokratie. Diagnosen und Reformvorschläge. Frankfurt a. M.: Campus.

Offe, Claus, und Ulrich K. Preuss. 1991. Democratic institutions and moral resources. In Political theory today, Hrsg. David Held. Cambridge: Cambridge University Press.

Pateman, Carole. 1970. Participation and democratic theory. London: Cambridge University Press.

Paxton, Pamela, Kenneth A. Bollen, Deborah M. Lee, und HyoJoung Kim. 2003. A half- century of suffrage: New data and a comparative analysis. Comparative International Development 38 (1): 93-122.

Pharr, Susan J., und Robert D. Putnam. 2000. Disaffected democracies. What's troubling the trilateral countries? Princeton: Princeton University Press. 
Plattner, Marc F. 2004. The quality of democracy: A skeptical afterword. Journal of Democracy 15 (4): $106-110$.

Popper, Karl. 1992 [1957]. Die offene Gesellschaft und ihre Feinde. Tübingen: Siebeck.

Powell, G. Bingham. 2004. Political representation in comparative politics. Annual Review of Political Science 7:273-296.

Putnam, Robert. 1993. Making democracy work. Princeton: Princeton University Press.

Rawls, John A. 1971. A theory of justice. Cambridge: Harvard University Press.

Rosendorff, B. Peter. 2004. Democracy and the supply of transparency. Paper presented at the annual meeting of the International Studies Association in Montreal, Quebec, Canada, March 172004.

Rousseau, Jean-Jacques. 1977 [1762]. Vom Gesellschaftsvertrag oder Grundsätze des Staatsrechts. Stuttgart: Reclam.

Rudolph, Thomas J., und Jillian Evans. 2005. Political trust, ideology, and public support for government spending. American Journal of Political Science 49 (3): 660-671.

Rueschemeyer, Dietrich. 2004. Adressing inequality. Journal of Democracy 15 (4): 76-90.

Sartori, Giovanni. 2006. Demokratietheorie. 3. Aufl. Darmstadt: WBG.

Saward, Michael. 1994. Democratic theory and indices of democratization. In Defining and measuring democracy, Hrsg. David Beetham, 6-24. London: Sage.

Saward, Michael. 1998. The terms of democracy. Cambridge: Polity Press.

Scharpf, Fritz. 1999. Regieren in Europa. Effektiv und demokratisch? Frankfurt a. M.: Campus.

Schiller, Theo. 1999. Prinzipien und Qualifizierungskriterien von Demokratie. In Perspektiven der Demokratie. Probleme und Chancen im Zeitalter der Globalisierung, Hrsg. Dirk Berg-Schlosser und Hans-Joachim Giegel, 28-56. Frankfurt a. M.: Campus.

Schmidt, Manfred G. 2000. Demokratietheorien: Eine Einführung. 3., überarb. und erw. Aufl. Opladen: Leske + Budrich.

Schneider, Aaron. 2003. Decentralization: Conceptualization and measurement. Studies in Comparative International Development 38 (3): 32-56.

Schumpeter, Joseph A. 1950. Kapitalismus, Sozialismus und Demokratie. 2., überarb. und erw. Ausgabe. Bern: Francke.

Sen, Amartya. 1979. Equality of what? The tanner lecture of human values. Stanford University.

Sen, Amartya. 1996. On the status of equality. Political Theory 24 (3): 394-400.

Sen, Amartya. 1997. From income inequality to economic inequality. Southern Economic Journal 64 (2): 383-401.

Steffani, Winfried. 1979. Parlamentarische und präsidentielle Demokratie. Opladen: Westdt.

Stiglitz, Joseph E. 1999. On liberty, the right to know, and public discourse: The role of transparency in public life. Oxford Amnesty Lecture, January 27, 1999.

Strom, Kaare. 1992. Democracy as political competition. American Behavioral Scientist 35 (4/5): 375-396.

Talmon, Jacob Leib. 1960. The origins of totalitarian democracy. New York: Praeger.

Tavits, Margit. 2006. Party system change: Testing a model of new party entry. Party Politics 12 (1): 99-119.

Teorell Jan. 2003. Linking social capital to political participation: voluntary associations and networks of recruitment in Sweden. Scandinavian Political Studies 26 (1): 49-66.

Teorell, Jan. 2006. Political participation and three theories of democracy: A research inventory and agenda. European Journal of Political Research 45 (5): 787-810.

Teorell, Jan, Paul Sum, und Mette Tobiasen. 2007. Participation and political equality. An assessment of large-scale democracy. In Citizenship and involvement in European democracies. A comparative analysis, Hrsg. Jan W. Van Deth, José Ramon Montero und Anders Westholm, 384-414. London: Routledge.

Tocqueville, Alexis de. 1997 [1835]. Über die Demokratie in Amerika. Stuttgart: Reclam.

Truman, David B. 1971 [1951]. The governmental process. New York: Knopf. 
Tsebelis, George. 1995. Decision making in political systems: Veto players in presidentialism, parliamentarism, multicameralism and multipartyism. British Journal of Political Science 25 (3): 289-325.

Urbinati, Nadja. 2010. Representative democracy and its critics. In The future of representative democracy, Hrsg. Sonia Alonso, John Keane und Wolfgang Merkel. Cambridge: Cambridge University Press (i.E.).

Urbinati, Nadia, und Mark E. Warren. 2008. The concept of representation in contemporary democratic theory. Annual Review of Political Science 11:387-412.

Vanhanen, Tatu. 1997. Prospects of democracy: A study of 172 countries. London: Routledge.

Vanhanen, Tatu. 2000. A new dataset for measuring democracy, 1810-1998. Journal of Peace Research 37 (2): 251-265.

Vanhanen, Tatu. 2003. Democratization. A comparative analysis of 170 countries. London: Routledge.

Vossenkuhl, Wilhelm. 1997. Gleichheit. In Lexikon der Ethik, Hrsg. Otfried Höffe, 108-110. München: Beck.

Warren, Mark E. 1996. What should we expect from more democracy? Radically democratic responses to politics. Political Theory 24 (2): 241-270.

Weber, Max. 1988 [1921]. Gesammelte Politische Schriften. Tübingen: Siebeck.

Welzel, Christian. 2000. Humanentwicklung und Demokratie: Welcher Index erfasst die ,humane Dimension der Demokratie am besten?. In Demokratiemessung. Konzepte und Befunde im internationalen Vergleich, Hrsg. Hans-Joachim Lauth, Gert Pickel und Christian Welzel, 132162. Opladen: Westdeutscher.

Wolbrecht, Christina, und David E. Campbell. 2007. Leading by example: Female members of parliament as political role models. American Journal of Political Science 51:921-39.

Wyckoff, Mikel L. 1987. Measures of attitudinal consistency as indicators of ideological sophistication: A reliability and validity assessment. The Journal of Politics 49 (1): 148-168.

Young, Iris Marion. 1999. State, civil society, and social justice. In Democracy's value, Hrsg. Ian Shapiro und Casiano Hacker-Cordon, 141-162. Cambridge: Cambridge University Press. 